\title{
NEWTON-LIKE INERTIAL DYNAMICS AND PROXIMAL ALGORITHMS GOVERNED BY MAXIMALLY MONOTONE OPERATORS
}

\author{
HEDY ATTOUCH* AND SZILÁRD CSABA LÁSZLÓ †
}

\begin{abstract}
The introduction of the Hessian damping in the continuous version of Nesterov's accelerated gradient method provides, by temporal discretization, fast proximal gradient algorithms where the oscillations are significantly attenuated. We will extend these results to the maximally monotone case. We rely on the technique introduced by Attouch-Peypouquet (Math. Prog. 2019), where the maximally monotone operator is replaced by its Yosida approximation with an appropriate adjustment of the regularization parameter. In a general Hilbert framework, we obtain the weak convergence of the iterates to equilibria, and the rapid convergence of the discrete velocities to zero. By specializing these algorithms to convex minimization, we obtain the convergence rate $o\left(1 / k^{2}\right)$ of the values, and the rapid convergence of the gradients towards zero.
\end{abstract}

Key words. Damped inertial dynamics; Hessian damping; large step proximal method; Lyapunov analysis; maximally monotone operators; Newton method; time-dependent viscosity; vanishing viscosity; Yosida regularization.

AMS subject classifications. 37N40, 46N10, 49M30, 65K05, 65K10, 90B50, 90C25.

Introduction. Let $\mathcal{H}$ be a real Hilbert space endowed with the scalar product $\langle\cdot, \cdot\rangle$ and norm $\|\cdot\|$. Given a general maximally monotone operator $A: \mathcal{H} \rightarrow 2^{\mathcal{H}}$, based on Newton's method, we want to design rapidly converging proximal algorithms to solve the monotone inclusion

$$
0 \in A x .
$$

Solving (0.1), i.e. find a zero of $A$, is a difficult problem of fundamental importance in optimization, equilibrium theory, economics and game theory, partial differential equations, statistics, among other subjects, (see for instance $[20,23,24,25,26,28,30]$ ). As a guide to our study, the algorithms will be derived from the temporal implicit discretization of the second-order differential equation

$$
(\mathrm{DIN}-\mathrm{AVD})_{\alpha, \beta} \quad \ddot{x}(t)+\frac{\alpha}{t} \dot{x}(t)+\beta \frac{d}{d t}\left(A_{\lambda(t)}(x(t))\right)+A_{\lambda(t)}(x(t))=0, \quad t>t_{0}>0,
$$

where $\alpha, \beta$ are positive damping parameters, and $J_{\lambda A}=(I+\lambda A)^{-1}, A_{\lambda}=\frac{1}{\lambda}\left(I-J_{\lambda A}\right)$ stand respectively for the resolvent of $A$ and the Yosida regularization of $A$ of index $\lambda>0$. According to the Lipschitz continuity property of $A_{\lambda},(\mathrm{DIN}-\mathrm{AVD})_{\alpha, \beta}$ is a well-posed evolution equation which enjoy nice asymptotic convergence properties. The object of our study is the "Proximal Regularized Inertial Newton Algorithm for Monotone operator", called (PRINAM) for short, and which can be viewed as a discrete temporal version of $(\mathrm{DIN}-\mathrm{AVD})_{\alpha, \beta}$. It is written as follows:

$$
\left\{\begin{array}{l}
y_{k}=\left(1-\beta\left(\frac{1}{\lambda_{k}}-\frac{1}{\lambda_{k-1}}\right)\right) x_{k}+\left(\alpha_{k}-\frac{\beta}{\lambda_{k-1}}\right)\left(x_{k}-x_{k-1}\right)+\frac{\beta}{\lambda_{k}} J_{\lambda_{k} A}\left(x_{k}\right)-\frac{\beta}{\lambda_{k-1}} J_{\lambda_{k-1} A}\left(x_{k-1}\right) \\
x_{k+1}=\frac{\lambda_{k+1}}{\lambda_{k+1}+s} y_{k}+\frac{s}{\lambda_{k+1}+s} J_{\left(\lambda_{k+1}+s\right) A}\left(y_{k}\right) .
\end{array}\right.
$$

*IMAG, Université Montpellier, CNRS, Place Eugène Bataillon, 34095 Montpellier CEDEX 5, France. E-mail: hedy.attouch@umontpellier.fr, Supported by COST Action: CA16228

${ }^{\dagger}$ Technical University of Cluj-Napoca, Department of Mathematics, Memorandumului 28, Cluj-Napoca, Romania, e-mail: szilard.laszlo@math.utcluj.ro., This work was supported by a grant of Ministry of Research and Innovation, CNCS - UEFISCDI, project number PN-III-P1-1.1-TE-2016-0266, Supported by COST Action: CA16228 
This algorithm includes both extrapolation and relaxation steps. Compared to the extrapolation step in the accelerated gradient method of Nesterov, its main characteristic is to include an additional correction term which is equal to the difference of the resolvents computed at two consecutive iterates. As a main result, we will prove that for an appropriate adjustment of the parameters, any sequence $\left(x_{k}\right)$ generated by this algorithm converges weakly to a zero of $A$. Moreover, when $A=\partial f$ specializes in the subdifferential of a convex lower semicontinuous function $f: \mathcal{H} \rightarrow \mathbb{R} \cup\{+\infty\}$, we obtain the convergence rate $o\left(\frac{1}{k^{2}}\right)$ of the values, and the fast convergence of the gradients towards zero. Our study is based on several recent advances in the study of inertial dynamics and algorithms for solving optimization problems and monotone inclusions. We describe them briefly in the following paragraphs. Our main contribution is to show how to put them together.

0.1. Asymptotic Vanishing Damping. The inertial system

$$
(\mathrm{AVD})_{\alpha} \quad \ddot{x}(t)+\frac{\alpha}{t} \dot{x}(t)+\nabla f(x(t))=0,
$$

was introduced in the context of convex optimization by Su-Boyd-Candès in [32]. For a general convex differentiable function $f$, it provides a continuous version of the accelerated gradient method of Nesterov. For $\alpha \geq 3$, each trajectory $x(\cdot)$ of $(\mathrm{AVD})_{\alpha}$ satisfies the asymptotic convergence rate of the values $f(x(t))-\inf _{\mathcal{H}} f=\mathcal{O}\left(1 / t^{2}\right)$. As a specific feature, the viscous damping coefficient $\frac{\alpha}{t}$ vanishes (tends to zero) as time $t$ goes to infinity, hence the terminology. The case $\alpha=3$, which corresponds to Nesterov's historical algorithm, is critical. In the case $\alpha=3$, the question of the convergence of the trajectories remains an open problem (except in one dimension where convergence holds [9]). For $\alpha>3$, it has been shown by Attouch-Chbani-Peypouquet-Redont [8] that each trajectory converges weakly to a minimizer. For $\alpha>3$, it is shown in [14] and [29] that the asymptotic convergence rate of the values is actually $o\left(1 / t^{2}\right)$. These rates are optimal, that is, they can be reached, or approached arbitrarily close. The corresponding inertial algorithms

$$
\left\{\begin{array}{l}
y_{k}=x_{k}+\left(1-\frac{\alpha}{k}\right)\left(x_{k}-x_{k-1}\right) \\
x_{k+1}=y_{k}-s \nabla f\left(y_{k}\right)
\end{array}\right.
$$

are in line with the Nesterov accelerated gradient method. They enjoy similar properties to the continuous case, see Chambolle-Dossal [22], and [6], [8], [14] for further results.

0.2. Hessian damping. The following inertial system

$$
\ddot{x}(t)+\frac{\alpha}{t} \dot{x}(t)+\beta \nabla^{2} f(x(t)) \dot{x}(t)+\nabla f(x(t))=0 .
$$

combines asymptotic vanishing damping with Hessian-driven damping. It was considered by AttouchPeypouquet-Redont in [15], (see also $[3,11]$ ). At first glance, the presence of the Hessian may seem to entail numerical difficulties. However, this is not the case as the Hessian intervenes in the form $\nabla^{2} f(x(t)) \dot{x}(t)$, which is nothing but the derivative with respect to time of the function $t \mapsto \nabla f(x(t))$. So, the temporal discretization of this dynamic provides first-order algorithms of the form

$$
\left\{\begin{array}{l}
y_{k}=x_{k}+\alpha_{k}\left(x_{k}-x_{k-1}\right)-\beta_{k}\left(\nabla f\left(x_{k}\right)-\nabla f\left(x_{k-1}\right)\right) \\
x_{k+1}=y_{k}-s \nabla f\left(y_{k}\right) .
\end{array}\right.
$$


As a specific feature, and by comparison with the accelerated gradient method of Nesterov, these algorithms contain a correction term which is equal to the difference of the gradients at two consecutive steps. While preserving the convergence properties of the Nesterov accelerated method, they provide fast convergence to zero of the gradients, and reduce the oscillatory aspects. Several recent studies have been devoted to this subject, see Attouch-Chbani-Fadili-Riahi [7], Bot-Csetnek-László [21], Kim [26], Lin-Jordan [27], Shi-Du-Jordan-Su [31].

0.3. Inertial dynamics and cocoercive operators. Let's come to the case of maximally monotone operators. Álvarez-Attouch [2] and Attouch-Maingé [10] studied the equation

$$
\ddot{x}(t)+\gamma \dot{x}(t)+A(x(t))=0
$$

when $A$ is a cocoercive ${ }^{1}$ (and hence maximally monotone) operator. Cocoercivity plays an important role in the study of (0.2), not only to ensure the existence of solutions, but also to analyze their long-term behavior. They showed that each trajectory of (0.2) converges weakly to a zero of $A$ if the cocoercivity parameter $\lambda$ and the damping coefficient $\gamma$ satisfy the inequality $\lambda \gamma^{2}>1$. Since $A_{\lambda}$ is $\lambda$-cocoercive and $A_{\lambda}^{-1}(0)=A^{-1}(0)$, we immediately deduce that, under the condition $\lambda \gamma^{2}>1$, given a general maximally monotone operator $A$, each trajectory of

$$
\ddot{x}(t)+\gamma \dot{x}(t)+A_{\lambda}(x(t))=0
$$

converges weakly to a zero of $A$. In the quest for a faster convergence, the analysis of

$$
(\mathrm{DIN}-\mathrm{AVD})_{\alpha, 0} \quad \ddot{x}(t)+\frac{\alpha}{t} \dot{x}(t)+A_{\lambda(t)}(x(t))=0, \quad t>t_{0}>0,
$$

leads to introduce a time-dependent parameter $\lambda(\cdot)$ satisfying $\lambda(t) \times \frac{\alpha^{2}}{t^{2}}>1$, see Attouch-Peypouquet [13]. Temporal discretization of this dynamic gives the Relaxed Inertial Proximal Algorithm

$$
(\mathrm{RIPA}) \quad\left\{\begin{array}{l}
y_{k}=x_{k}+\alpha_{k}\left(x_{k}-x_{k-1}\right) \\
x_{k+1}=\left(1-\rho_{k}\right) y_{k}+\rho_{k} J_{\mu_{k} A}\left(y_{k}\right)
\end{array}\right.
$$

whose convergence properties have been analyzed by Attouch-Peypouquet [13], Attouch-Cabot [5].

0.4. Link with Newton-like methods for solving monotone inclusions. Let us specify the link between our study and Newton's method for solving (0.1). To overcome the ill-posed character of the continuous Newton method, the following first-order evolution system was studied by Attouch-Svaiter (see [17]), for a general maximally monotone operator $A$

$$
\left\{\begin{array}{l}
v(t) \in A(x(t)) \\
\gamma(t) \dot{x}(t)+\beta \dot{v}(t)+v(t)=0
\end{array}\right.
$$

This system can be considered as a continuous version of the Levenberg-Marquardt method, which acts as a regularization of the Newton method. Remarkably, under a fairly general assumption on the regularization parameter $\gamma(t)$, this system is well posed and generates trajectories that converge

\footnotetext{
${ }^{1} A: \mathcal{H} \rightarrow \mathcal{H}$ is $\lambda$-cocoercive $\left(\lambda\right.$ is a positive parameter) if for all $x, y \in \mathcal{H}\langle A y-A x, y-x\rangle \geq \lambda\|A y-A x\|^{2}$
} 
weakly to equilibria. Parallel results have been obtained for the associated proximal algorithms obtained by implicit temporal discretization, see [1], [12], [16], [19]. Formally, this system writes as

$$
\gamma(t) \dot{x}(t)+\beta \frac{d}{d t}(A(x(t)))+A(x(t))=0 .
$$

Thus, (DIN - AVD $)_{\alpha, \beta}$ can be considered as an inertial and regularized version of this system.

0.5. Organization of the paper. The (PRINAM) algorithm, which is our main subject, is studied in section 1 . Then, in section 2 we will examine the case $A=\partial f$ where $f: \mathcal{H} \rightarrow \mathbb{R} \cup\{+\infty\}$ is a convex lower semicontinuous function. Finally, we outline some perspectives.

1. Convergence of the associated proximal relaxed algorithm. The (PRINAM) algorithm will be introduced by implicit temporal discretization of $(\mathrm{DIN}-\mathrm{AVD})_{\alpha, \beta}$. In view of the Lipschitz continuity property of $A_{\lambda}$, the explicit discretization might work well too. In fact, the implicit discretization tends to follow the continuous-time trajectories more closely. In addition, the implicit and explicit discretizations have a comparable iteration complexity.

1.1. Regularized Inertial Proximal Algorithms. Take a fixed time step $h>0$, and set $t_{k}=k h, x_{k}=x\left(t_{k}\right), \lambda_{k}=\lambda\left(t_{k}\right)$. Consider the implicit finite-difference scheme for (DIN - AVD) ${ }_{\alpha, \beta}$

$$
\frac{1}{h^{2}}\left(x_{k+1}-2 x_{k}+x_{k-1}\right)+\frac{\alpha}{k h^{2}}\left(x_{k}-x_{k-1}\right)+\frac{\beta}{h}\left(A_{\lambda_{k}}\left(x_{k}\right)-A_{\lambda_{k-1}}\left(x_{k-1}\right)\right)+A_{\lambda_{k+1}}\left(x_{k+1}\right)=0 .
$$

with centered second-order variation. After expanding (1.1), we obtain

$$
x_{k+1}+h^{2} A_{\lambda_{k+1}}\left(x_{k+1}\right)=x_{k}+\left(1-\frac{\alpha}{k}\right)\left(x_{k}-x_{k-1}\right)-\beta h\left(A_{\lambda_{k}}\left(x_{k}\right)-A_{\lambda_{k-1}}\left(x_{k-1}\right)\right) .
$$

Set $s=h^{2}$. Keeping the notation $\beta$ for $\beta h$, and setting $\alpha_{k}:=\left(1-\frac{\alpha}{k}\right)$, we have

$$
x_{k+1}+s A_{\lambda_{k+1}}\left(x_{k+1}\right)=y_{k},
$$

where

$$
y_{k}:=x_{k}+\alpha_{k}\left(x_{k}-x_{k-1}\right)-\beta\left(A_{\lambda_{k}}\left(x_{k}\right)-A_{\lambda_{k-1}}\left(x_{k-1}\right)\right) .
$$

From (1.3) we get

$$
x_{k+1}=\left(I+s A_{\lambda_{k+1}}\right)^{-1}\left(y_{k}\right),
$$

where $\left(I+s A_{\lambda_{k+1}}\right)^{-1}$ is the resolvent of index $s>0$ of the maximally monotone operator $A_{\lambda_{k+1}}$. Putting (1.4) and (1.5) together, we obtain the following algorithm

$$
\left\{\begin{aligned}
y_{k} & =x_{k}+\alpha_{k}\left(x_{k}-x_{k-1}\right)-\beta\left(A_{\lambda_{k}}\left(x_{k}\right)-A_{\lambda_{k-1}}\left(x_{k-1}\right)\right) \\
x_{k+1} & =\left(I+s A_{\lambda_{k+1}}\right)^{-1}\left(y_{k}\right) .
\end{aligned}\right.
$$

Let us give some equivalent formulations of this algorithm. According to the resolvent equation (formulated as a semi-group property) $\left(A_{\lambda}\right)_{s}=A_{\lambda+s}$, we have

$$
\left(I+s A_{\lambda}\right)^{-1}=I-s\left(A_{\lambda}\right)_{s}=I-s A_{\lambda+s} .
$$


Thus, we obtain the following formulation, which makes use of the Yosida approximations of $A$.

$$
\left\{\begin{array}{l}
y_{k}=x_{k}+\alpha_{k}\left(x_{k}-x_{k-1}\right)-\beta\left(A_{\lambda_{k}}\left(x_{k}\right)-A_{\lambda_{k-1}}\left(x_{k-1}\right)\right) \\
x_{k+1}=y_{k}-s A_{\lambda_{k+1}+s}\left(y_{k}\right) .
\end{array}\right.
$$

According to $A_{\lambda}=\frac{1}{\lambda}\left(I-J_{\lambda A}\right)$, let us reformulate (1.7) using the resolvents of $A$. We have

$$
\begin{array}{r}
A_{\lambda_{k}}\left(x_{k}\right)-A_{\lambda_{k-1}}\left(x_{k-1}\right)=\frac{1}{\lambda_{k}} x_{k}-\frac{1}{\lambda_{k-1}} x_{k-1}-\left(\frac{1}{\lambda_{k}} J_{\lambda_{k} A}\left(x_{k}\right)-\frac{1}{\lambda_{k-1}} J_{\lambda_{k-1} A}\left(x_{k-1}\right)\right) \\
=\frac{1}{\lambda_{k-1}}\left(x_{k}-x_{k-1}\right)+\left(\frac{1}{\lambda_{k}}-\frac{1}{\lambda_{k-1}}\right) x_{k}-\left(\frac{1}{\lambda_{k}} J_{\lambda_{k} A}\left(x_{k}\right)-\frac{1}{\lambda_{k-1}} J_{\lambda_{k-1} A}\left(x_{k-1}\right)\right) \\
y_{k}-s A_{\lambda_{k+1}+s}\left(y_{k}\right)=y_{k}-\frac{s}{\lambda_{k+1}+s}\left(y_{k}-J_{\left(\lambda_{k+1}+s\right) A}\left(y_{k}\right)\right)=\frac{\lambda_{k+1}}{\lambda_{k+1}+s} y_{k}+\frac{s}{\lambda_{k+1}+s} J_{\left(\lambda_{k+1}+s\right) A}\left(y_{k}\right) .
\end{array}
$$

This gives the "Proximal Regularized Inertial Newton Algorithm for Monotone operator", called (PRINAM) for short. It is formulated below in terms of the resolvents of $A$.

\begin{tabular}{|} 
(PRINAM) \\
Take $x_{0} \in \mathcal{H}, x_{1} \in \mathcal{H}$ \\
Step $k:\left\{\begin{array}{r}y_{k}=\left(1-\beta\left(\frac{1}{\lambda_{k}}-\frac{1}{\lambda_{k-1}}\right)\right) x_{k}+\left(\alpha_{k}-\frac{\beta}{\lambda_{k-1}}\right)\left(x_{k}-x_{k-1}\right) \\
+\beta\left(\frac{1}{\lambda_{k}} J_{\lambda_{k} A}\left(x_{k}\right)-\frac{1}{\lambda_{k-1}} J_{\lambda_{k-1} A}\left(x_{k-1}\right)\right) \\
x_{k+1}=\frac{\lambda_{k+1}}{\lambda_{k+1}+s} y_{k}+\frac{s}{\lambda_{k+1}+s} J_{\left(\lambda_{k+1}+s\right) A}\left(y_{k}\right) .\end{array}\right.$
\end{tabular}

We are now in position to prove the main result of this section, namely:

Theorem 1.1. Let $A: \mathcal{H} \rightarrow 2^{\mathcal{H}}$ be a maximally monotone operator such that $S=A^{-1}(0) \neq \emptyset$. Consider the algorithm (PRINAM) where, for all $k \geq 0, \quad \alpha_{k}=\frac{t_{k}-1}{t_{k+1}}, t_{k}=r k+q, r>0, q \in \mathbb{R}$ and

$$
\lambda_{k}=\lambda k^{2} \text { with } \lambda>\frac{(2 \beta+s)^{2} r^{2}}{s} \text {. }
$$

Then, for any sequences $\left(x_{k}\right),\left(y_{k}\right)$ generated by (PRINAM), the following properties are satisfied:

i) The speed tends to zero, and we have the following estimates

$$
\begin{gathered}
\left\|x_{k+1}-x_{k}\right\|=\mathcal{O}\left(\frac{1}{k}\right) \text { as } k \rightarrow+\infty, \quad \sum_{k} k\left\|x_{k}-x_{k-1}\right\|^{2}<+\infty \\
\left\|A_{\lambda_{k}}\left(x_{k}\right)\right\|=o\left(\frac{1}{k^{2}}\right) \text { as } k \rightarrow+\infty, \quad \sum_{k} k^{3}\left\|A_{\lambda_{k}}\left(x_{k}\right)\right\|^{2}<+\infty .
\end{gathered}
$$

ii) The sequence $\left(x_{k}\right)$ converges weakly to some $\hat{x} \in S$, as $k \rightarrow+\infty$.

iii) The sequence $\left(y_{k}\right)$ converges weakly to $\hat{x} \in S$, as $k \rightarrow+\infty$.

Precisely, $\left\|y_{k}-x_{k}\right\|=\mathcal{O}\left(\frac{1}{k}\right)$, as $k \rightarrow+\infty$, and so $y_{k}-x_{k}$ converges strongly to zero. 
1.2. Geometric interpretation. In algorithm (PRINAM), the proximal parameter $\lambda_{k}$ tends to infinity in a controlled way, namely $\lambda_{k}=\lambda k^{2}$ with $\lambda$ sufficiently large. This property balances the vanishing property of the damping coefficient. As a classical property of the resolvents ([18, Theorem 23.44]), for any $x \in \mathcal{H}, J_{\lambda A} x \rightarrow \operatorname{proj}_{S}(x)$ as $\lambda \rightarrow+\infty$, where $S$ is the set of zeros of $A$. Thus the algorithm writes

$$
x_{k+1}=\theta_{k} y_{k}+\left(1-\theta_{k}\right) J_{\left(\lambda_{k+1}+s\right) A}\left(y_{k}\right)=y_{k}+\frac{s}{\lambda_{k+1}+s}\left(J_{\left(\lambda_{k+1}+s\right) A}\left(y_{k}\right)-y_{k}\right)
$$

with $\lambda_{k} \sim+\infty, \theta_{k}=\frac{\lambda_{k+1}}{\lambda_{k+1}+s} \sim 1, \frac{s}{\lambda_{k+1}+s} \sim 0, J_{\left(\lambda_{k+1}+s\right) A}\left(y_{k}\right) \sim \operatorname{proj}_{S}\left(y_{k}\right)$ as $k \rightarrow+\infty$. At step $k$, after reaching $y_{k}$, the direction $J_{\left(\lambda_{k+1}+s\right) A}\left(y_{k}\right)-y_{k} \sim \operatorname{proj}_{S}\left(y_{k}\right)-y_{k}$ is well oriented in the direction of $S$, but we are allowed to take only a small step in this direction. This is illustrated in figure 1.1 .

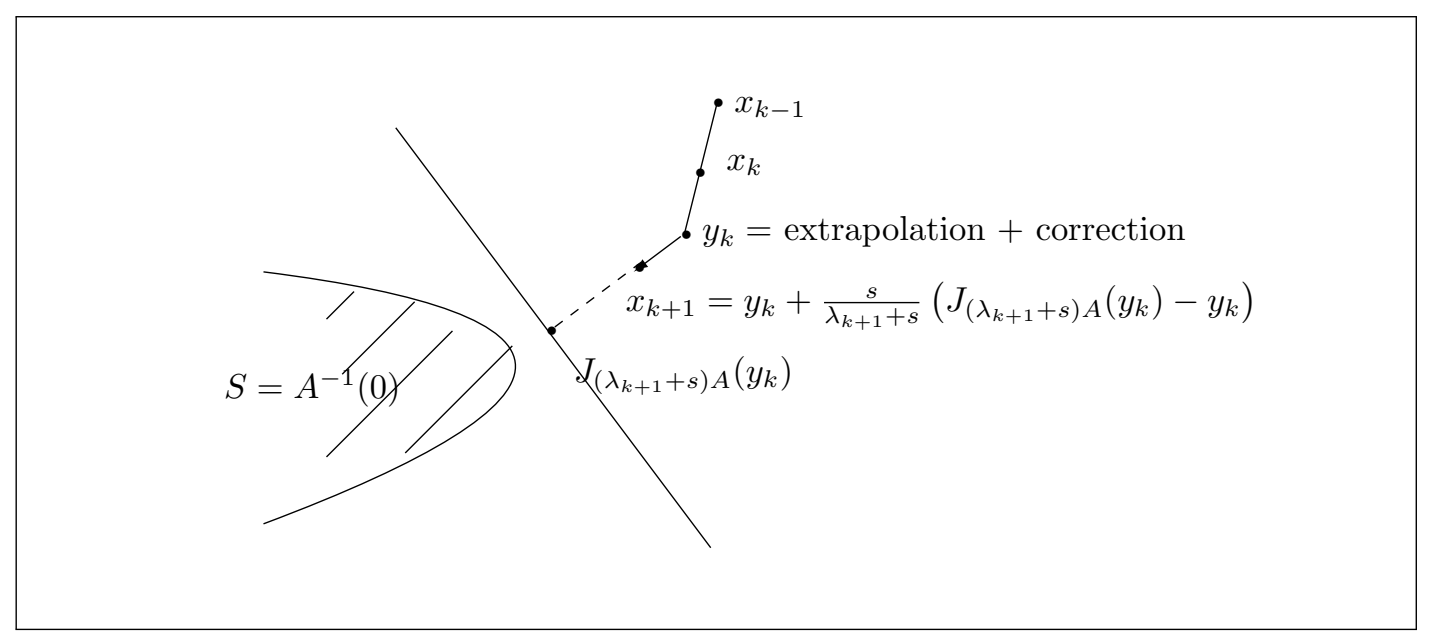

Fig. 1.1: (PRINAM) algorithm

REMARK 1. Following [5]-[6], we could develop our theory with a general sequence $\left(\alpha_{k}\right)$ of extrapolation coefficients which satisfy $0 \leq \alpha_{k} \leq 1$. A particularly interesting situation is the case $\alpha_{k} \rightarrow 1$, which corresponds to the asymptotic vanishing damping in the associated dynamic system. The sequence $\left(t_{k}\right)$ which is linked to the sequence $\left(\alpha_{k}\right)$ by the relation

$$
\alpha_{k}=\frac{t_{k}-1}{t_{k+1}}
$$

plays a central role. To simplify the presentation, in Theorem 1.1 we limit our study to the case

$$
t_{k}=r k+q, r>0, q \in \mathbb{R}
$$

which contains most interesting situations. In particular, when $\alpha_{k}=1-\frac{\alpha}{k}$, we have $t_{k}=\frac{k-1}{\alpha-1}$, which corresponds to $r=\frac{1}{\alpha-1}, q=-\frac{1}{\alpha-1}$. The critical value $\alpha=3$ corresponds to $r=\frac{1}{2}$. 


\subsection{Proof of Theorem 1.1.}

The discrete energy. Take $z \in S$. For each $k \geq 1$, let us define the discrete energy

$$
\begin{aligned}
\mathcal{E}_{a, b}^{k} & :=a t_{k-1}\left\langle A_{\lambda_{k-1}}\left(x_{k-1}\right), x_{k-1}-z\right\rangle+\frac{1}{2}\left\|b\left(x_{k-1}-z\right)+t_{k}\left(x_{k}-x_{k-1}+s A_{\lambda_{k}}\left(x_{k}\right)\right)\right\|^{2} \\
& +\frac{b(1-b)}{2}\left\|x_{k-1}-z\right\|^{2} .
\end{aligned}
$$

We will show that by adjusting the real parameters $a$ and $b$, the following Lyapunov property is satisfied: there exist $\epsilon_{1}, \epsilon_{2}>0$ and an index $N \in \mathbb{N}$ such that for all $k \geq N$

$$
\mathcal{E}_{a, b}^{k+1}-\mathcal{E}_{a, b}^{k}+\epsilon_{1} k^{3}\left\|A_{\lambda_{k}}\left(x_{k}\right)\right\|^{2}+\epsilon_{2} k\left\|x_{k}-x_{k-1}\right\|^{2} \leq 0 .
$$

Specifically, in what follows, we will take

$$
\begin{aligned}
& 0<b<1 \text { and } b \beta<a<b \beta+b s, \text { whenever } \beta>0 \\
& a=0 \text { for } \beta=0 .
\end{aligned}
$$

For each $k \geq 1$, briefly write $\mathcal{E}_{a, b}$ as follows

$$
\mathcal{E}_{a, b}^{k}=a t_{k-1}\left\langle A_{\lambda_{k-1}}\left(x_{k-1}\right), x_{k-1}-z\right\rangle+\frac{1}{2}\left\|v_{k}\right\|^{2}+\frac{b(1-b)}{2}\left\|x_{k-1}-z\right\|^{2},
$$

with

$$
v_{k}:=b\left(x_{k-1}-z\right)+t_{k}\left(x_{k}-x_{k-1}+s A_{\lambda_{k}}\left(x_{k}\right)\right) .
$$

Using successively the definition of $v_{k}$, (1.3) (1.4), and (1.8) we obtain

$$
\begin{aligned}
v_{k+1} & =b\left(x_{k}-z\right)+t_{k+1}\left(x_{k+1}-x_{k}+s A_{\lambda_{k+1}}\left(x_{k+1}\right)\right) \\
& =b\left(x_{k}-z\right)+t_{k+1}\left(y_{k}-x_{k}\right) \\
& =b\left(x_{k}-z\right)+t_{k+1}\left(\alpha_{k}\left(x_{k}-x_{k-1}\right)-\beta\left(A_{\lambda_{k}}\left(x_{k}\right)-A_{\lambda_{k-1}}\left(x_{k-1}\right)\right)\right) \\
& =b\left(x_{k}-z\right)+\left(t_{k}-1\right)\left(x_{k}-x_{k-1}\right)-\beta t_{k+1}\left(A_{\lambda_{k}}\left(x_{k}\right)-A_{\lambda_{k-1}}\left(x_{k-1}\right)\right) .
\end{aligned}
$$

Further, $v_{k}$ can be written as

$$
v_{k}=b\left(x_{k}-z\right)+\left(t_{k}-b\right)\left(x_{k}-x_{k-1}\right)+s t_{k} A_{\lambda_{k}}\left(x_{k}\right) .
$$

Therefore, for all $k \geq 1$, we have

$$
\begin{aligned}
\frac{1}{2}\left\|v_{k+1}\right\|^{2}-\frac{1}{2}\left\|v_{k}\right\|^{2}=\frac{1}{2} & \left\|b\left(x_{k}-z\right)+\left(t_{k}-1\right)\left(x_{k}-x_{k-1}\right)-\beta t_{k+1}\left(A_{\lambda_{k}}\left(x_{k}\right)-A_{\lambda_{k-1}}\left(x_{k-1}\right)\right)\right\|^{2} \\
& -\frac{1}{2}\left\|b\left(x_{k}-z\right)+\left(t_{k}-b\right)\left(x_{k}-x_{k-1}\right)+s t_{k} A_{\lambda_{k}}\left(x_{k}\right)\right\|^{2} \\
=\frac{1}{2} & \left(\left(t_{k}-1\right)^{2}-\left(t_{k}-b\right)^{2}\right)\left\|x_{k}-x_{k-1}\right\|^{2} \\
& +\frac{1}{2}\left(\beta^{2} t_{k+1}^{2}-s^{2} t_{k}^{2}\right)\left\|A_{\lambda_{k}}\left(x_{k}\right)\right\|^{2}-\beta^{2} t_{k+1}^{2}\left\langle A_{\lambda_{k}}\left(x_{k}\right), A_{\lambda_{k-1}}\left(x_{k-1}\right)\right\rangle \\
& +\frac{1}{2} \beta^{2} t_{k+1}^{2}\left\|A_{\lambda_{k-1}}\left(x_{k-1}\right)\right\|^{2}+b(b-1)\left\langle x_{k}-x_{k-1}, x_{k}-z\right\rangle \\
& -b\left(\beta t_{k+1}+s t_{k}\right)\left\langle A_{\lambda_{k}}\left(x_{k}\right), x_{k}-z\right\rangle+b \beta t_{k+1}\left\langle A_{\lambda_{k-1}}\left(x_{k-1}\right), x_{k}-z\right\rangle \\
& -\left(\beta t_{k+1}\left(t_{k}-1\right)+s t_{k}\left(t_{k}-b\right)\right)\left\langle A_{\lambda_{k}}\left(x_{k}\right), x_{k}-x_{k-1}\right\rangle \\
& +\beta t_{k+1}\left(t_{k}-1\right)\left\langle A_{\lambda_{k-1}}\left(x_{k-1}\right), x_{k}-x_{k-1}\right\rangle .
\end{aligned}
$$


According to the elementary identities

$$
\begin{aligned}
& b(b-1)\left\langle x_{k}-x_{k-1}, x_{k}-z\right\rangle=b(b-1)\left\|x_{k}-x_{k-1}\right\|^{2}+b(b-1)\left\langle x_{k}-x_{k-1}, x_{k-1}-z\right\rangle \\
& b \beta t_{k+1}\left\langle A_{\lambda_{k-1}}\left(x_{k-1}\right), x_{k}-z\right\rangle=b \beta t_{k+1}\left\langle A_{\lambda_{k-1}}\left(x_{k-1}\right), x_{k}-x_{k-1}\right\rangle+b \beta t_{k+1}\left\langle A_{\lambda_{k-1}}\left(x_{k-1}\right), x_{k-1}-z\right\rangle
\end{aligned}
$$

formula (1.15) becomes

$$
\begin{aligned}
\frac{1}{2}\left\|v_{k+1}\right\|^{2}-\frac{1}{2}\left\|v_{k}\right\|^{2} & =\frac{1}{2}(b-1)\left(2 t_{k}+b-1\right)\left\|x_{k}-x_{k-1}\right\|^{2} \\
& +\frac{1}{2}\left(\beta^{2} t_{k+1}^{2}-s^{2} t_{k}^{2}\right)\left\|A_{\lambda_{k}}\left(x_{k}\right)\right\|^{2}-\beta^{2} t_{k+1}^{2}\left\langle A_{\lambda_{k}}\left(x_{k}\right), A_{\lambda_{k-1}}\left(x_{k-1}\right)\right\rangle \\
& +\frac{1}{2} \beta^{2} t_{k+1}^{2}\left\|A_{\lambda_{k-1}}\left(x_{k-1}\right)\right\|^{2}+b(b-1)\left\langle x_{k}-x_{k-1}, x_{k-1}-z\right\rangle \\
& -b\left(\beta t_{k+1}+s t_{k}\right)\left\langle A_{\lambda_{k}}\left(x_{k}\right), x_{k}-z\right\rangle+b \beta t_{k+1}\left\langle A_{\lambda_{k-1}}\left(x_{k-1}\right), x_{k-1}-z\right\rangle \\
& -\left(\beta t_{k+1}\left(t_{k}-1\right)+s t_{k}\left(t_{k}-b\right)\right)\left\langle A_{\lambda_{k}}\left(x_{k}\right), x_{k}-x_{k-1}\right\rangle \\
& +\beta t_{k+1}\left(t_{k}+b-1\right)\left\langle A_{\lambda_{k-1}}\left(x_{k-1}\right), x_{k}-x_{k-1}\right\rangle .
\end{aligned}
$$

Moreover, we have for all $k \geq 1$

$$
\begin{aligned}
& \frac{b(1-b)}{2}\left\|x_{k}-z\right\|^{2}-\frac{b(1-b)}{2}\left\|x_{k-1}-z\right\|^{2} \\
& \quad=\frac{b(1-b)}{2}\left\|\left(x_{k}-x_{k-1}\right)+\left(x_{k-1}-z\right)\right\|^{2}-\frac{b(1-b)}{2}\left\|x_{k-1}-z\right\|^{2} \\
& \quad=\frac{b(1-b)}{2}\left\|x_{k}-x_{k-1}\right\|^{2}+b(1-b)\left\langle x_{k}-x_{k-1}, x_{k-1}-z\right\rangle
\end{aligned}
$$

By combining the above results (the terms $\left\langle x_{k}-x_{k-1}, x_{k-1}-z\right\rangle$ cancel out), we get for all $k \geq 1$

$$
\begin{aligned}
& \mathcal{E}_{a, b}^{k+1}- \mathcal{E}_{a, b}^{k}=\left(a t_{k}-b\left(\beta t_{k+1}+s t_{k}\right)\right)\left\langle A_{\lambda_{k}}\left(x_{k}\right), x_{k}-z\right\rangle+\left(b \beta t_{k+1}-a t_{k-1}\right)\left\langle A_{\lambda_{k-1}}\left(x_{k-1}\right), x_{k-1}-z\right\rangle \\
&+\frac{1}{2}\left(\beta^{2} t_{k+1}^{2}-s^{2} t_{k}^{2}\right)\left\|A_{\lambda_{k}}\left(x_{k}\right)\right\|^{2}-\beta^{2} t_{k+1}^{2}\left\langle A_{\lambda_{k}}\left(x_{k}\right), A_{\lambda_{k-1}}\left(x_{k-1}\right)\right\rangle+\frac{1}{2} \beta^{2} t_{k+1}^{2}\left\|A_{\lambda_{k-1}}\left(x_{k-1}\right)\right\|^{2} \\
&-\left(\beta t_{k+1}\left(t_{k}-1\right)+s t_{k}\left(t_{k}-b\right)\right)\left\langle A_{\lambda_{k}}\left(x_{k}\right), x_{k}-x_{k-1}\right\rangle \\
&(1.18)+\beta t_{k+1}\left(t_{k}+b-1\right)\left\langle A_{\lambda_{k-1}}\left(x_{k-1}\right), x_{k}-x_{k-1}\right\rangle+\frac{1}{2}(b-1)\left(2 t_{k}-1\right)\left\|x_{k}-x_{k-1}\right\|^{2} .
\end{aligned}
$$

According to the assumptions (1.11) and (1.12) on the parameters $a$ and $b$, there exists $k_{1} \geq 1$ such that for all $k \geq k_{1}$

$$
a t_{k}-b\left(\beta t_{k+1}+s t_{k}\right)<0 \text { and } b \beta t_{k+1}-a t_{k-1} \leq 0
$$

where in the last relation the equality holds only in case $a=0, \beta=0$. According to the cocoerciveness of $A_{\lambda_{k}}$ and $A_{\lambda_{k-1}}$ and $z \in S$, we deduce from the above inequalities that, for all $k \geq k_{1}$

$$
\begin{gathered}
\left(a t_{k}-b\left(\beta t_{k+1}+s t_{k}\right)\right)\left\langle A_{\lambda_{k}}\left(x_{k}\right), x_{k}-z\right\rangle \leq\left(a t_{k}-b\left(\beta t_{k+1}+s t_{k}\right)\right) \lambda_{k}\left\|A_{\lambda_{k}}\left(x_{k}\right)\right\|^{2} \\
\left(b \beta t_{k+1}-a t_{k-1}\right)\left\langle A_{\lambda_{k-1}}\left(x_{k-1}\right), x_{k-1}-z\right\rangle \leq\left(b \beta t_{k+1}-a t_{k-1}\right) \lambda_{k-1}\left\|A_{\lambda_{k-1}}\left(x_{k-1}\right)\right\|^{2} .
\end{gathered}
$$


Therefore, (1.18) yields, for all $k \geq k_{1}$

$$
\begin{aligned}
\mathcal{E}_{a, b}^{k+1}-\mathcal{E}_{a, b}^{k} & \leq\left(\left(a t_{k}-b\left(\beta t_{k+1}+s t_{k}\right)\right) \lambda_{k}+\frac{1}{2}\left(\beta^{2} t_{k+1}^{2}-s^{2} t_{k}^{2}\right)\right)\left\|A_{\lambda_{k}}\left(x_{k}\right)\right\|^{2} \\
& +\left(\left(b \beta t_{k+1}-a t_{k-1}\right) \lambda_{k-1}+\frac{1}{2} \beta^{2} t_{k+1}^{2}\right)\left\|A_{\lambda_{k-1}}\left(x_{k-1}\right)\right\|^{2} \\
& +\frac{1}{2}(b-1)\left(2 t_{k}-1\right)\left\|x_{k}-x_{k-1}\right\|^{2}-\beta^{2} t_{k+1}^{2}\left\langle A_{\lambda_{k}}\left(x_{k}\right), A_{\lambda_{k-1}}\left(x_{k-1}\right)\right\rangle \\
& -\left(\beta t_{k+1}\left(t_{k}-1\right)+s t_{k}\left(t_{k}-b\right)\right)\left\langle A_{\lambda_{k}}\left(x_{k}\right), x_{k}-x_{k-1}\right\rangle \\
& +\beta t_{k+1}\left(t_{k}+b-1\right)\left\langle A_{\lambda_{k-1}}\left(x_{k-1}\right), x_{k}-x_{k-1}\right\rangle .
\end{aligned}
$$

Further, for all $p_{1}, p_{2}>0$ and $k \geq k_{1}$ we have the elementary inequalities

$$
\begin{aligned}
& -\beta^{2} t_{k+1}^{2}\left\langle A_{\lambda_{k}}\left(x_{k}\right), A_{\lambda_{k-1}}\left(x_{k-1}\right)\right\rangle \leq \frac{\beta^{2}}{2} t_{k+1}^{2}\left(\left\|A_{\lambda_{k}}\left(x_{k}\right)\right\|^{2}+\left\|A_{\lambda_{k-1}}\left(x_{k-1}\right)\right\|^{2}\right) ; \\
& -\left(\beta t_{k+1}\left(t_{k}-1\right)+s t_{k}\left(t_{k}-b\right)\right)\left\langle A_{\lambda_{k}}\left(x_{k}\right), x_{k}-x_{k-1}\right\rangle \\
& \quad \leq\left|\beta t_{k+1}\left(t_{k}-1\right)+s t_{k}\left(t_{k}-b\right)\right|\left(p_{1} k\left\|A_{\lambda_{k}}\left(x_{k}\right)\right\|^{2}+\frac{1}{4 p_{1} k}\left\|x_{k}-x_{k-1}\right\|^{2}\right) \\
& \quad \beta t_{k+1}\left(t_{k}+b-1\right)\left\langle A_{\lambda_{k-1}}\left(x_{k-1}\right), x_{k}-x_{k-1}\right\rangle \\
& \quad \leq\left|\beta t_{k+1}\left(t_{k}+b-1\right)\right|\left(p_{2} k\left\|A_{\lambda_{k-1}}\left(x_{k-1}\right)\right\|^{2}+\frac{1}{4 p_{2} k}\left\|x_{k}-x_{k-1}\right\|^{2}\right) .
\end{aligned}
$$

Combining (1.19) with (1.20)-(1.21)-(1.22), we deduce that, for all $k \geq k_{1}$

$$
\mathcal{E}_{a, b}^{k+1}-\mathcal{E}_{a, b}^{k} \leq P(k)\left\|A_{\lambda_{k}}\left(x_{k}\right)\right\|^{2}+Q(k)\left\|A_{\lambda_{k-1}}\left(x_{k-1}\right)\right\|^{2}+R(k)\left\|x_{k}-x_{k-1}\right\|^{2},
$$

where

$$
\begin{aligned}
& P(k)=\left(a t_{k}-b\left(\beta t_{k+1}+s t_{k}\right)\right) \lambda_{k}+\frac{1}{2}\left(\beta^{2} t_{k+1}^{2}-s^{2} t_{k}^{2}\right)+\frac{\beta^{2}}{2} t_{k+1}^{2}+\left|\beta t_{k+1}\left(t_{k}-1\right)+s t_{k}\left(t_{k}-b\right)\right| p_{1} k ; \\
& Q(k)=\left(b \beta t_{k+1}-a t_{k-1}\right) \lambda_{k-1}+\frac{1}{2} \beta^{2} t_{k+1}^{2}+\frac{\beta^{2}}{2} t_{k+1}^{2}+\left|\beta t_{k+1}\left(t_{k}+b-1\right)\right| p_{2} k ; \\
& R(k)=\frac{1}{2}(b-1)\left(2 t_{k}-1\right)+\frac{\left|\beta t_{k+1}\left(t_{k}-1\right)+s t_{k}\left(t_{k}-b\right)\right|}{4 p_{1} k}+\frac{\left|\beta t_{k+1}\left(t_{k}+b-1\right)\right|}{4 p_{2} k} .
\end{aligned}
$$

Elementary computation gives the following asymptotic developments

$$
\begin{aligned}
& P(k)=\left((a-b \beta-b s) r \lambda+(\beta+s) r^{2} p_{1}\right) k^{3}+r_{1}(k) \\
& Q(k)=\left((b \beta-a) r \lambda+\beta r^{2} p_{2}\right) k^{3}+r_{2}(k) \\
& R(k)=\left((b-1) r+\frac{(\beta+s) r^{2}}{4 p_{1}}+\frac{\beta r^{2}}{4 p_{2}}\right) k+r_{3}(k),
\end{aligned}
$$

where $r_{1}(k), r_{2}(k)=\mathcal{O}\left(k^{2}\right)$ and $r_{3}(k)=\mathcal{O}(1)$ as $k \rightarrow+\infty$. Let us adjust the parameters by taking

$$
\begin{aligned}
& b=\frac{1}{2} \in(0,1), \quad a=\frac{\beta(\beta+s)}{2 \beta+s} \in(b \beta, b(\beta+s)) \text { whenever } \beta>0 \\
& b=\frac{1}{2} \text { and } a=0 \text { whenever } \beta=0 .
\end{aligned}
$$


In this last case $Q \equiv 0$. For the rest of the proof we do not need to distinguish the cases $\beta>0$ and $\beta=0$. Further, take

$$
p_{1}=p_{2}=\frac{\lambda s}{2 \epsilon(2 \beta+s) r} \text { with } 1<\epsilon<\frac{\lambda s}{(2 \beta+s)^{2} r^{2}} .
$$

This is possible, thanks to our basic assumption on $\lambda$, namely $\lambda>\frac{(2 \beta+s)^{2} r^{2}}{s}$. With this choice of parameters, we have for all $k \geq k_{1}$

$$
\begin{aligned}
& P(k)=\frac{(\beta+s) s r \lambda}{2(2 \beta+s)}\left(\frac{1}{\epsilon}-1\right) k^{3}+r_{1}(k), \\
& Q(k)=\frac{\beta s r \lambda}{2(2 \beta+s)}\left(\frac{1}{\epsilon}-1\right) k^{3}+r_{2}(k), \\
& R(k)=\left(-\frac{1}{2} r+\frac{(2 \beta+s)^{2} \epsilon r^{3}}{2 s \lambda}\right) k+r_{3}(k) .
\end{aligned}
$$

Since $\epsilon>1$, we have $\frac{(\beta+s) s r \lambda}{2(2 \beta+s)}\left(\frac{1}{\epsilon}-1\right)<0$ and $\frac{\beta s r \lambda}{2(2 \beta+s)}\left(\frac{1}{\epsilon}-1\right) \leq 0$.

Since $\epsilon<\frac{\lambda s}{(2 \beta+s)^{2} r^{2}}$, we have $-\frac{1}{2} r+\frac{(2 \beta+s)^{2} \epsilon r^{3}}{2 s \lambda}<0$. So, there exists $\epsilon_{1}, \epsilon_{2}>0$ such that

$$
\frac{(\beta+s) s r \lambda}{2(2 \beta+s)}\left(\frac{1}{\epsilon}-1\right)+\epsilon_{1}<0 \text { and }\left(-\frac{1}{2} r+\frac{(2 \beta+s)^{2} \epsilon r^{3}}{2 s \lambda}\right)+\epsilon_{2}<0 .
$$

According to the above inequalities, (1.23) leads to

$$
\begin{aligned}
\mathcal{E}_{a, b}^{k+1}-\mathcal{E}_{a, b}^{k} & +\epsilon_{1} k^{3}\left\|A_{\lambda_{k}}\left(x_{k}\right)\right\|^{2}+\epsilon_{2} k\left\|x_{k}-x_{k-1}\right\|^{2} \\
& \leq\left(\left(\frac{(\beta+s) s r \lambda}{2(2 \beta+s)}\left(\frac{1}{\epsilon}-1\right)+\epsilon_{1}\right) k^{3}+r_{1}(k)\right)\left\|A_{\lambda_{k}}\left(x_{k}\right)\right\|^{2} \\
& +\left(\frac{\beta s r \lambda}{2(2 \beta+s)}\left(\frac{1}{\epsilon}-1\right) k^{3}+r_{2}(k)\right)\left\|A_{\lambda_{k-1}}\left(x_{k-1}\right)\right\|^{2} \\
& +\left(\left(-\frac{1}{2} r+\frac{(2 \beta+s)^{2} \epsilon r^{3}}{2 s \lambda}+\epsilon_{2}\right) k+r_{3}(k)\right)\left\|x_{k}-x_{k-1}\right\|^{2} .
\end{aligned}
$$

Take $N \geq k_{1}$ such that, for all $k \geq N$

$$
\begin{aligned}
& \left(\frac{(\beta+s) s r \lambda}{2(2 \beta+s)}\left(\frac{1}{\epsilon}-1\right)+\epsilon_{1}\right) k^{3}+r_{1}(k) \leq 0, \\
& \frac{\beta s r \lambda}{2(2 \beta+s)}\left(\frac{1}{\epsilon}-1\right) k^{3}+r_{2}(k) \leq 0 \\
& \left(-\frac{1}{2} r+\frac{(2 \beta+s)^{2} \epsilon r^{3}}{2 s \lambda}+\epsilon_{2}\right) k+r_{3}(k) \leq 0 .
\end{aligned}
$$

Then, for all $k \geq N$

$$
\mathcal{E}_{a, b}^{k+1}-\mathcal{E}_{a, b}^{k}+\epsilon_{1} k^{3}\left\|A_{\lambda_{k}}\left(x_{k}\right)\right\|^{2}+\epsilon_{2} k\left\|x_{k}-x_{k-1}\right\|^{2} \leq 0 .
$$


Estimates. According to (1.25), the sequence of non-negative numbers $\left(\mathcal{E}_{a, b}^{k}\right)_{k \in \mathbb{N}}$ is non-increasing, and therefore converges. In particular, it is bounded. From this, and by adding the inequalities (1.25) we obtain

$$
\begin{aligned}
& \sup _{k} t_{k}\left\langle A_{\lambda_{k}}\left(x_{k}\right), x_{k}-z\right\rangle<+\infty, \\
& \sup _{k}\left\|b\left(x_{k}-z\right)+t_{k+1}\left(x_{k+1}-x_{k}+s A_{\lambda_{k+1}}\left(x_{k+1}\right)\right)\right\|^{2}<+\infty, \\
& \sup _{k}\left\|x_{k}-z\right\|^{2}<+\infty \\
& \sum_{k=0}^{+\infty} k^{3}\left\|A_{\lambda_{k}}\left(x_{k}\right)\right\|^{2}<+\infty, \\
& \sum_{k=1}^{+\infty} k\left\|x_{k}-x_{k-1}\right\|^{2}<+\infty .
\end{aligned}
$$

Since the general term of a convergent series goes to zero, we deduce from (1.29) that

$$
\left\|A_{\lambda_{k}}\left(x_{k}\right)\right\|=o\left(\frac{1}{k^{\frac{3}{2}}}\right) \text { as } k \rightarrow+\infty .
$$

In fact, we will get better estimates a little further. According to (1.28), the sequence $\left(\left\|x_{k}-z\right\|\right)$ is bounded, and so is the sequence $\left(x_{k}\right)$. Combining the above results with (1.27), we deduce that

$$
\left\|x_{k}-x_{k-1}\right\|=\mathcal{O}\left(\frac{1}{k}\right) \text { as } k \rightarrow+\infty .
$$

From $\left(x_{k}\right)$ bounded, and $A_{\lambda_{k}} \frac{1}{\lambda_{k}}$ - Lipschitz continuous, we obtain the existence of $M>0$ such that

$$
\left\|\lambda_{k} A_{\lambda_{k}}\left(x_{k}\right)\right\|=\left\|\lambda_{k} A_{\lambda_{k}}\left(x_{k}\right)-\lambda_{k} A_{\lambda_{k}}(z)\right\| \leq \lambda_{k} \frac{1}{\lambda_{k}}\left\|x_{k}-z\right\| \leq M
$$

which yields

$$
\left\|A_{\lambda_{k}}\left(x_{k}\right)\right\|=\mathcal{O}\left(\frac{1}{k^{2}}\right) \text { as } k \rightarrow+\infty .
$$

Let us show the following better estimate which will play a key role in the rest of the proof

$$
\left\|A_{\lambda_{k}}\left(x_{k}\right)\right\|=o\left(\frac{1}{k^{2}}\right) \text { as } k \rightarrow+\infty .
$$

To obtain it, we follow the line of proof of [13, Theorem 3.6]. From Lemma A.4 [13], for all $k \geq 1$

$$
\left\|\lambda_{k} A_{\lambda_{k}}\left(x_{k}\right)-\lambda_{k-1} A_{\lambda_{k-1}}\left(x_{k-1}\right)\right\| \leq 2\left\|x_{k}-x_{k-1}\right\|+2\left\|x_{k}-z\right\| \frac{\left|\lambda_{k}-\lambda_{k-1}\right|}{\lambda_{k}} .
$$

According to $\left\|x_{k}-x_{k-1}\right\|=\mathcal{O}\left(\frac{1}{k}\right)$ as $k \rightarrow+\infty,\left(x_{k}\right)$ is bounded, and $\lambda_{k}=\lambda k^{2}$ we conclude that there exists $C>0$ such that

$$
\left\|\lambda_{k} A_{\lambda_{k}}\left(x_{k}\right)-\lambda_{k-1} A_{\lambda_{k-1}}\left(x_{k-1}\right)\right\| \leq \frac{C}{k}, \text { for all } k \geq 1
$$


According to (1.33) and (1.36), we deduce that

$$
\begin{aligned}
& \left|\left\|\lambda_{k} A_{\lambda_{k}}\left(x_{k}\right)\right\|^{2}-\left\|\lambda_{k-1} A_{\lambda_{k-1}}\left(x_{k-1}\right)\right\|^{2}\right| \\
& =\left(\left\|\lambda_{k} A_{\lambda_{k}}\left(x_{k}\right)\right\|+\left\|\lambda_{k-1} A_{\lambda_{k-1}}\left(x_{k-1}\right)\right\|\right)\left|\left\|\lambda_{k} A_{\lambda_{k}}\left(x_{k}\right)\right\|-\left\|\lambda_{k-1} A_{\lambda_{k-1}}\left(x_{k-1}\right)\right\|\right| \\
& \leq 2 M\left\|\lambda_{k} A_{\lambda_{k}}\left(x_{k}\right)-\lambda_{k-1} A_{\lambda_{k-1}}\left(x_{k-1}\right)\right\| \leq \frac{2 M C}{k} \text {, for all } k \geq 1 .
\end{aligned}
$$

Consequently, by using (1.29) we get

$$
\begin{aligned}
& \sum_{k}\left|\left\|\lambda_{k} A_{\lambda_{k}}\left(x_{k}\right)\right\|^{4}-\left\|\lambda_{k-1} A_{\lambda_{k-1}}\left(x_{k-1}\right)\right\|^{4}\right| \\
& =\sum_{k}\left(\left\|\lambda_{k} A_{\lambda_{k}}\left(x_{k}\right)\right\|^{2}+\left\|\lambda_{k-1} A_{\lambda_{k-1}}\left(x_{k-1}\right)\right\|^{2}\right)\left|\left\|\lambda_{k} A_{\lambda_{k}}\left(x_{k}\right)\right\|^{2}-\left\|\lambda_{k-1} A_{\lambda_{k-1}}\left(x_{k-1}\right)\right\|^{2}\right| \\
& \leq \sum_{k} \frac{2 M C \lambda^{2} k^{4}}{k}\left\|A_{\lambda_{k}}\left(x_{k}\right)\right\|^{2}+\sum_{k} \frac{2 M C \lambda^{2}(k-1)^{4}}{k}\left\|A_{\lambda_{k-1}}\left(x_{k-1}\right)\right\|^{2}<+\infty .
\end{aligned}
$$

From this, by a telescopic argument we conclude that $\lim _{k \rightarrow+\infty}\left\|\lambda_{k} A_{\lambda_{k}}\left(x_{k}\right)\right\|^{4}$ exists.

But then $\lim _{k \rightarrow+\infty}\left\|\lambda_{k} A_{\lambda_{k}}\left(x_{k}\right)\right\|^{2}$ and $\lim _{k \rightarrow+\infty}\left\|\lambda_{k} A_{\lambda_{k}}\left(x_{k}\right)\right\|$ also exist. Set

$$
\lim _{k \rightarrow+\infty} k^{4}\left\|A_{\lambda_{k}}\left(x_{k}\right)\right\|^{2}:=L \geq 0
$$

According to (1.29) we will have

$$
\sum_{k} \frac{1}{k}\left(k^{4}\left\|A_{\lambda_{k}}\left(x_{k}\right)\right\|^{2}\right)=\sum_{k} k^{3}\left\|A_{\lambda_{k}}\left(x_{k}\right)\right\|^{2}<+\infty,
$$

which implies that $L=0$. Hence, $\lim _{k \rightarrow+\infty} k^{2}\left\|A_{\lambda_{k}}\left(x_{k}\right)\right\|=0$, that is

$$
\left\|A_{\lambda_{k}}\left(x_{k}\right)\right\|=o\left(\frac{1}{k^{2}}\right) \text { as } k \rightarrow+\infty \text {. }
$$

Convergence of $\left(x_{k}\right)$. Using the Opial's lemma, let us prove that the sequence $\left(x_{k}\right)$ converges weakly towards an element of $S$. Take $z \in S$, and consider the anchor sequence $\left(h_{k}\right)$ defined by $h_{k}=\frac{1}{2}\left\|x_{k}-z\right\|^{2}$ for $k \geq 1$. Elementary algebra gives

$$
h_{k+1}-h_{k}=\frac{1}{2}\left\|x_{k+1}-x_{k}\right\|^{2}+\left\langle x_{k+1}-x_{k}, x_{k}-z\right\rangle .
$$

According to (1.6) we have

$$
\begin{aligned}
\left\langle x_{k+1}-x_{k}, x_{k}-z\right\rangle & =\left\langle y_{k}-x_{k}-s A_{\lambda_{k+1}}\left(x_{k+1}\right), x_{k}-z\right\rangle \\
& =\alpha_{k}\left\langle x_{k}-x_{k-1}, x_{k}-z\right\rangle-\beta\left\langle A_{\lambda_{k}}\left(x_{k}\right)-A_{\lambda_{k-1}}\left(x_{k-1}\right), x_{k}-z\right\rangle \\
& -s\left\langle A_{\lambda_{k+1}}\left(x_{k+1}\right), x_{k}-z\right\rangle .
\end{aligned}
$$

Let us examine the terms involved in the above equality. We have

$$
\left\langle x_{k}-x_{k-1}, x_{k}-z\right\rangle=\left\|x_{k}-x_{k-1}\right\|^{2}+\left\langle x_{k}-x_{k-1}, x_{k-1}-z\right\rangle=h_{k}-h_{k-1}+\frac{1}{2}\left\|x_{k}-x_{k-1}\right\|^{2} .
$$


and

$$
-s\left\langle A_{\lambda_{k+1}}\left(x_{k+1}\right), x_{k}-z\right\rangle=s\left\langle A_{\lambda_{k+1}}\left(x_{k+1}\right), x_{k+1}-x_{k}\right\rangle-s\left\langle A_{\lambda_{k+1}}\left(x_{k+1}\right), x_{k+1}-z\right\rangle .
$$

Combining these relations with (1.38) and (1.39), and neglecting the term $-s\left\langle A_{\lambda_{k+1}}\left(x_{k+1}\right), x_{k+1}-z\right\rangle$ which is non-positive, we obtain

$$
\begin{aligned}
h_{k+1}-h_{k} & \leq \alpha_{k}\left(h_{k}-h_{k-1}\right)+\frac{1}{2}\left\|x_{k+1}-x_{k}\right\|^{2}+\frac{\alpha_{k}}{2}\left\|x_{k}-x_{k-1}\right\|^{2} \\
& -\beta\left\langle A_{\lambda_{k}}\left(x_{k}\right)-A_{\lambda_{k-1}}\left(x_{k-1}\right), x_{k}-z\right\rangle+s\left\langle A_{\lambda_{k+1}}\left(x_{k+1}\right), x_{k+1}-x_{k}\right\rangle .
\end{aligned}
$$

According to $\left\|x_{k}-z\right\|$ bounded, $\left\|A_{\lambda_{k+1}}\left(x_{k+1}\right)\right\|=o\left(\frac{1}{k^{2}}\right)$ and $\left\|x_{k+1}-x_{k}\right\|=\mathcal{O}\left(\frac{1}{k}\right)$, we obtain the existence of a constant $M>0$ such that

$$
\begin{aligned}
h_{k+1}-h_{k} & \leq \alpha_{k}\left(h_{k}-h_{k-1}\right)+\frac{1}{2}\left\|x_{k+1}-x_{k}\right\|^{2}+\frac{\alpha_{k}}{2}\left\|x_{k}-x_{k-1}\right\|^{2} \\
& +M\left\|A_{\lambda_{k}}\left(x_{k}\right)-A_{\lambda_{k-1}}\left(x_{k-1}\right)\right\|+M \frac{1}{k^{3}} .
\end{aligned}
$$

In addition, by (1.36) and by the fact that $\lambda_{k}=\lambda k^{2}$, we get

$$
\left\|\lambda k^{2} A_{\lambda_{k}}\left(x_{k}\right)-\lambda(k-1)^{2} A_{\lambda_{k-1}}\left(x_{k-1}\right)\right\| \leq \frac{C}{k}, \text { for all } k \geq 1
$$

Equivalently,

$$
\left\|(2 \lambda k-\lambda) A_{\lambda_{k}}\left(x_{k}\right)+\lambda(k-1)^{2}\left(A_{\lambda_{k}}\left(x_{k}\right)-A_{\lambda_{k-1}}\left(x_{k-1}\right)\right)\right\| \leq \frac{C}{k}, \text { for all } k \geq 1 .
$$

Using again that $\left\|A_{\lambda_{k+1}}\left(x_{k+1}\right)\right\|=o\left(\frac{1}{k^{2}}\right)$, we deduce that, for some $K>0$

$$
\left\|A_{\lambda_{k}}\left(x_{k}\right)-A_{\lambda_{k-1}}\left(x_{k-1}\right)\right\| \leq \frac{K}{k^{3}} .
$$

Therefore, (1.41) leads to

$$
h_{k+1}-h_{k} \leq \alpha_{k}\left(h_{k}-h_{k-1}\right)+\frac{1}{2}\left\|x_{k+1}-x_{k}\right\|^{2}+\frac{\alpha_{k}}{2}\left\|x_{k}-x_{k-1}\right\|^{2}+M K \frac{1}{k^{3}}+M \frac{1}{k^{3}} .
$$

Let us analyze this inequality with the help of the Lemma A.1. Set

$$
\omega_{k}:=\frac{1}{2}\left\|x_{k+1}-x_{k}\right\|^{2}+\frac{\alpha_{k}}{2}\left\|x_{k}-x_{k-1}\right\|^{2}+M K \frac{1}{k^{3}}+M \frac{1}{k^{3}} .
$$

As a direct result of the estimates we have already obtained, we have $\sum_{k} t_{k+1} \omega_{k}<+\infty$. Therefore, by applying Lemma A.1 to the sequence $a_{k}=\left[h_{k}-h_{k-1}\right]_{+}$we obtain

$$
\sum_{k}\left[h_{k}-h_{k-1}\right]_{+}<+\infty
$$

Since $h_{k}$ is nonnegative, this property classically gives the existence of $\lim _{k \rightarrow+\infty} h_{k}$, and hence of the existence of $\lim _{k \rightarrow+\infty}\left\|x_{k}-z\right\|$. This shows item (i) of the Opial lemma. 
It remains to show that every weak cluster point of the sequence $\left(x_{k}\right)$ belongs to $S$. Let $x^{*}$ be a weak cluster point of $\left(x_{k}\right)$ and consider a subsequence $\left(x_{k_{n}}\right)$ of $\left(x_{k}\right)$, such that $x_{k_{n}} \rightarrow x^{*}, n \rightarrow+\infty$. According to (1.37) we have

$$
\lim _{k \rightarrow+\infty} \lambda_{k} A_{\lambda_{k}}\left(x_{k}\right)=0 .
$$

Now use $A_{\lambda_{k_{n}}}\left(x_{k_{n}}\right) \in A\left(J_{\lambda_{k_{n}} A}\left(x_{k_{n}}\right)\right)$. Equivalently,

$$
A_{\lambda_{k_{n}}}\left(x_{k_{n}}\right) \in A\left(x_{k_{n}}-\lambda_{k_{n}} A_{\lambda_{k_{n}}}\left(x_{k_{n}}\right)\right) .
$$

According to the demi-closedness of the graph of $A$, passing to the limit in (1.44) gives

$$
0 \in A\left(x^{*}\right) .
$$

According to Opial's lemma, we finally obtain that $\left(x_{k}\right)$ converges weakly to an element $\hat{x}$ in $S$. Finally, by definition of $y_{k}$, we have

$$
y_{k}-x_{k}=\alpha_{k}\left(x_{k}-x_{k-1}\right)-\beta\left(A_{\lambda_{k}}\left(x_{k}\right)-A_{\lambda_{k-1}}\left(x_{k-1}\right)\right),
$$

which, combined with (1.32) and (1.42), gives

$$
\left\|y_{k}-x_{k}\right\|=\mathcal{O}\left(\frac{1}{k}\right), \text { as } k \rightarrow+\infty .
$$

Therefore, $\left(y_{k}\right)$ also converges weakly towards the same element $\hat{x}$ in $S$.

1.4. Comparison with related algorithms. By taking $\beta=0$ and $r=\frac{1}{\alpha-1}, q=-\frac{1}{\alpha-1}$ in (PRINAM), we obtain the algorithm (RIPA) considered by Attouch-Peypouquet in [13]. This algorithm and its convergence properties are recalled below

$$
\left\{\begin{aligned}
y_{k} & =x_{k}+\left(1-\frac{\alpha}{k}\right)\left(x_{k}-x_{k-1}\right) \\
x_{k+1} & =\frac{\lambda_{k}}{\lambda_{k}+s} y_{k}+\frac{s}{\lambda_{k}+s} J_{\left(\lambda_{k}+s\right) A}\left(y_{k}\right) .
\end{aligned}\right.
$$

Theorem (Attouch-Peypouquet, [13]) Let $A: \mathcal{H} \rightarrow 2^{\mathcal{H}}$ be a maximally monotone operator with $S=A^{-1}(0) \neq \emptyset$. Let $\left(x_{k}\right)$ be a sequence generated by (RIPA) where $s>0, \alpha>2$ and for all $k \geq 1$

$$
\lambda_{k}=\lambda k^{2} \text { for some } \lambda>\frac{s}{\alpha(\alpha-2)} .
$$

Then, the sequences $\left(x_{k}\right)$ and $\left(y_{k}\right)$ converge weakly, as $k \rightarrow+\infty$, to some $\hat{x} \in S$.

In addition, $\left\|x_{k+1}-x_{k}\right\|=\mathcal{O}\left(\frac{1}{k}\right)$ as $k \rightarrow+\infty$, and $\sum_{k} k\left\|x_{k}-x_{k-1}\right\|^{2}<+\infty$.

A natural question is to compare (PRINAM) to (RIPA), and show what the introduction of the correcting term in (PRINAM) $(\beta>0)$ brings. We emphasize that, for small $\beta$ and $r=\frac{1}{\alpha-1}$, the lower bound for $\lambda$ obtained in Theorem 1.1 namely $\lambda>\frac{(2 \beta+s)^{2} r^{2}}{s}$ is better than the lower bound obtained in the above result, namely $\lambda>\frac{s}{\alpha(\alpha-2)}$. Further, in (PRINAM) the more general condition $\alpha>1$ is allowed. As a model example of a maximally monotone operator which is not the subdifferential of a convex function, consider $A: \mathbb{R}^{2} \rightarrow \mathbb{R}^{2}$ given for any $x=(\xi, \eta) \in \mathbb{R}^{2}$ by

$$
A(\xi, \eta)=(-\eta, \xi) .
$$

$A$ is a skew symmetric linear operator whose single zero is $x^{*}=(0,0)$. An easy computation shows that $A$ and $A_{\lambda}$ can be identified respectively with the matrices 


$$
A=\left(\begin{array}{cc}
0 & -1 \\
1 & 0
\end{array}\right) \quad A_{\lambda}=\left(\begin{array}{cc}
\frac{\lambda}{1+\lambda^{2}} & \frac{-1}{1+\lambda^{2}} \\
\frac{1}{1+\lambda^{2}} & \frac{\lambda}{1+\lambda^{2}}
\end{array}\right) .
$$

Let's compare (PRINAM) and (RIPA) by considering different instances of the parameters involved.

- Take $\alpha=3$, then $\alpha=11$ in (RIPA), which corresponds to respectively $t_{k}=0.5 k-0.5$, $t_{k}=0.1 k-0.1,(r=0.5, r=0.1, q=-0.5, q=-0.1)$, in (PRINAM).

- Take $\lambda_{k}=\lambda k^{2}$ with $\lambda$ chosen as follows:

To satisfy the condition $\lambda>\frac{s}{\alpha(\alpha-2)}$ in (RIPA), we take $\lambda=1.01 \frac{s}{\alpha(\alpha-2)}$ in (RIPA).

To satisfy the condition $\lambda>\frac{(2 \beta+s)^{2} r^{2}}{s}$ in (PRINAM), we take $\lambda=1.01 \frac{(2 \beta+s)^{2} r^{2}}{s}$.

- For the step size $s$, we consider the following instances: $s \in\{0.01,0.1,0.5,1\}$. For (PRINAM) we consider the values $\beta \in\{0,0.1 s, 0.25 s, 0.35 s, 0.5 s\}$.

To start the algorithm we take $x_{0}=(1,-1), x_{1}=(-1,1)$. We run the algorithms until the iteration error $\left\|x_{k}-x^{*}\right\|$ reaches the value $10^{-5}$. The results are depicted at Figure 1.2 1.2a-1.2d. The horizontal and vertical axis show respectively the number of iterations and the value of the error $\left\|x_{k}-x^{*}\right\|$. Despite the fact that it is difficult to draw general conclusions from a single numerical experiment, the above result shows the numerical interest of the introduction of the correcting term $(\beta>0)$, and also that the step size $s$ must be taken not too large (therefore not remaining too far from the continuous dynamics). We only report here numerical examples where $s$ is relatively small, for large values of $s$ the convergence properties are less good. Further, $r$ should be taken small (or $\alpha$ large) in order to obtain fast convergence.

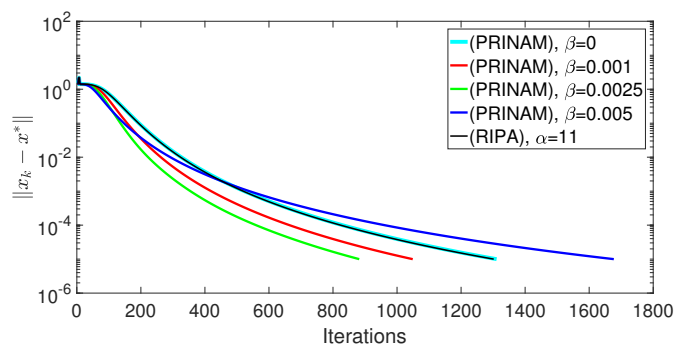

(a) $s=0.01, r=0.1, q=-0.1$

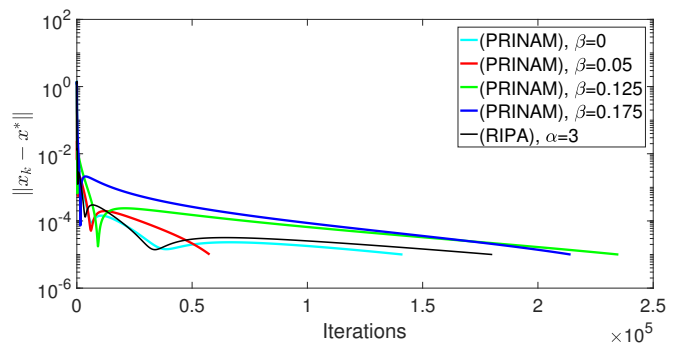

(c) $s=0.5, r=0.5, q=-0.5$

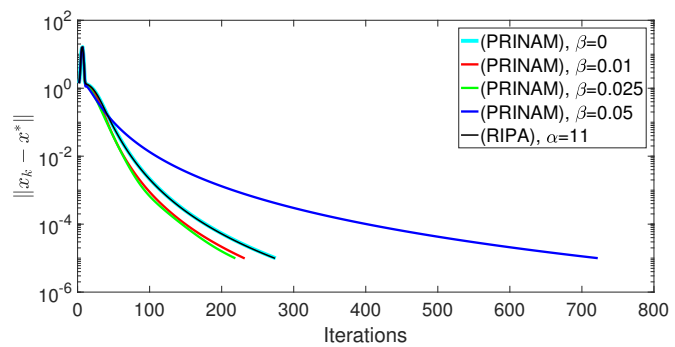

(b) $s=0.1, r=0.1, q=-0.1$

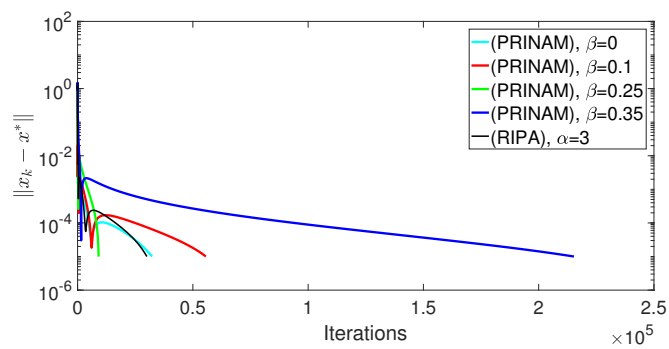

(d) $s=1, r=0.5, q=-0.5$

Fig. 1.2: Iteration error $\left\|x_{k}-x^{*}\right\|$ for different instances of (PRINAM) and (RIPA) 
2. The convex case. Let us specialize the previous results to the case of convex minimization, and show the rapid convergence of values. Given a lower semi-continuous convex and proper function $f: \mathcal{H} \rightarrow \mathbb{R} \cup\{+\infty\}$ such that $\operatorname{argmin} f \neq \emptyset$, we consider the minimization problem

$$
(\mathcal{P}) \inf _{x \in \mathcal{H}} f(x)
$$

Fermat's rule states that $x$ is a global minimum of $f$ if and only if

$$
0 \in \partial f(x)
$$

Therefore, $(\mathcal{P})$ is equivalent to the monotone inclusion problem $(2.1)$, and $\operatorname{argmin} f=(\partial f)^{-1}(0)$. The Yosida approximation of $\partial f$ is equal to the gradient of the Moreau envelope of $f$ : for any $\lambda>0$

$$
(\partial f)_{\lambda}=\nabla f_{\lambda}
$$

Recall that $f_{\lambda}: \mathcal{H} \rightarrow \mathbb{R}$, is a $\mathcal{C}^{1,1}$ function, which is defined by: for any $x \in \mathcal{H}$

$$
f_{\lambda}(x)=\inf _{\xi \in \mathcal{H}}\left\{f(\xi)+\frac{1}{2 \lambda}\|x-\xi\|^{2}\right\}
$$

When we specialize the (PRINAM) algorithm in the case $A=\partial f$, we obtain

\section{(PRINAM)-convex}

Take $x_{0} \in \mathcal{H}, x_{1} \in \mathcal{H}$

Step $k:\left\{\begin{array}{l}y_{k}=x_{k}+\alpha_{k}\left(x_{k}-x_{k-1}\right)-\beta\left(\nabla f_{\lambda_{k}}\left(x_{k}\right)-\nabla f_{\lambda_{k-1}}\left(x_{k-1}\right)\right) \\ x_{k+1}=y_{k}-s \nabla f_{\lambda_{k+1}+s}\left(y_{k}\right) .\end{array}\right.$

The next result is a direct consequence of Theorem 1.1

THEOREM 2.1. Let $\left(x_{k}\right),\left(y_{k}\right)$ be sequences generated by the algorithm (PRINAM)-convex. Assume that $\alpha_{k}=\frac{t_{k}-1}{t_{k+1}}, t_{k}=r k+q, r>0, q \in \mathbb{R}$ and for all $k \geq 0$

Then, the following properties are satisfied:

$$
\lambda_{k}=\lambda k^{2}, \text { with } \lambda>\frac{(2 \beta+s)^{2} r^{2}}{s} \text {. }
$$

i) The speed tends to zero, and we have the following estimates

$$
\begin{array}{cc}
\text { (pointwise) } \quad\left\|x_{k+1}-x_{k}\right\|=\mathcal{O}\left(\frac{1}{k}\right), \quad\left\|\nabla f_{\lambda_{k}}\left(x_{k}\right)\right\|=o\left(\frac{1}{k^{2}}\right) \quad \text { as } k \rightarrow+\infty . \\
\text { (summation) } \quad \sum_{k} k\left\|x_{k}-x_{k-1}\right\|^{2}<+\infty, \quad \sum_{k} k^{3}\left\|\nabla f_{\lambda_{k}}\left(x_{k}\right)\right\|^{2}<+\infty .
\end{array}
$$

ii) The sequences $\left(x_{k}\right),\left(y_{k}\right)$ converge weakly, as $k \rightarrow+\infty$, to some $\hat{x} \in \operatorname{argmin} f$.

iii) We have the convergence rates of the values: as $k \rightarrow+\infty$

$$
f_{\lambda_{k}}\left(x_{k}\right)-\min f=o\left(\frac{1}{k^{2}}\right) \quad \text { and } f\left(\operatorname{prox}_{\lambda_{k} f}\left(x_{k}\right)\right)-\min f=o\left(\frac{1}{k^{2}}\right) .
$$

In addition, $\left\|\operatorname{prox}_{\lambda_{k} f}\left(x_{k}\right)-x_{k}\right\| \rightarrow 0$ as $k \rightarrow+\infty$. 
Proof. (i) and (ii) follow directly from Theorem 1.1 applied to the operator $\partial f$ and using (2.2). (iii). Take $x^{*} \in \operatorname{argmin} f$. From the gradient inequality, and $\left(x_{k}\right)$ is bounded, for all $k \geq 0$ we have

$$
\begin{aligned}
f_{\lambda_{k}}\left(x_{k}\right)-\min _{\mathcal{H}} f & =f_{\lambda_{k}}\left(x_{k}\right)-f_{\lambda_{k}}\left(x^{*}\right) \leq\left\langle\nabla f_{\lambda_{k}}\left(x_{k}\right), x_{k}-x^{*}\right\rangle \\
& \leq\left\|\nabla f_{\lambda_{k}}\left(x_{k}\right)\right\|\left\|x_{k}-x^{*}\right\| \leq M\left\|\nabla f_{\lambda_{k}}\left(x_{k}\right)\right\| .
\end{aligned}
$$

Combining the above relation with $\left\|\nabla f_{\lambda_{k}}\left(x_{k}\right)\right\|=o\left(\frac{1}{k^{2}}\right)$ as $k \rightarrow+\infty$ (see (1.37)), we obtain

$$
f_{\lambda_{k}}\left(x_{k}\right)-\min _{\mathcal{H}} f=o\left(\frac{1}{k^{2}}\right) \text { as } k \rightarrow+\infty .
$$

By definition of $f_{\lambda_{k}}$ and of the proximal mapping, we have

$$
f_{\lambda_{k}}\left(x_{k}\right)-\min _{\mathcal{H}} f=f\left(\operatorname{prox}_{\lambda_{k} f}\left(x_{k}\right)\right)-\min _{\mathcal{H}} f+\frac{1}{2 \lambda_{k}}\left\|x_{k}-\operatorname{prox}_{\lambda_{k} f}\left(x_{k}\right)\right\|^{2} .
$$

Combining (2.3) with (2.4), we obtain

$$
f\left(\operatorname{prox}_{\lambda_{k} f}\left(x_{k}\right)\right)-\min _{\mathcal{H}} f=o\left(\frac{1}{k^{2}}\right) \text { as } k \rightarrow+\infty, \quad \lim _{k \rightarrow+\infty} k^{2} \frac{1}{2 \lambda_{k}}\left\|x_{k}-\operatorname{prox}_{\lambda_{k} f}\left(x_{k}\right)\right\|^{2}=0 .
$$

The above relation leads to $\lim _{k \rightarrow+\infty}\left\|x_{k}-\operatorname{prox}_{\lambda_{k} f}\left(x_{k}\right)\right\|=0$, which completes the proof.

REMARK 2. When $A=\partial f, f$ convex, we have additional tools, such as the gradient inequality. We will show in the following theorem that, in this case, some assumptions can be weakened. When $\beta=0$, we will obtain fast convergence of the values for $\lambda_{k}=\lambda k^{t}, t \geq 0, \lambda>0$, that is, under the mild assumption that the sequence $\left(\lambda_{k}\right)$ is nonincreasing. Further, fast convergence can be obtained in the general case $\beta>0$ provided that the sequence $\left(\lambda_{k}\right)$ is constant or $\lambda_{k}=\lambda k^{t}, t>1, \lambda>0$.

THEOREM 2.2. Let $\left(x_{k}\right),\left(y_{k}\right)$ be sequences generated by the algorithm (PRINAM)-convex. Assume that $\alpha_{k}=\frac{t_{k}-1}{t_{k+1}}$ and $\lambda_{k}=\lambda k^{t}, t \geq 0$ for all $k \geq 0$, further $t_{k}=r k+q, r \in\left(0, \frac{1}{2}\right), q \in \mathbb{R}$, that is, there exists $k_{1} \geq 0$ and $m \in(0,1)$ such that

$$
t_{k} \geq 1, m t_{k+1} \geq t_{k+1}^{2}-t_{k}^{2}, \text { for all } k \geq k_{1} .
$$

i) Assume that one of the following conditions hold.

(a) $\beta=0, t \geq 0$.

(b) $\beta>0, t=0, s>2 \beta$.

(c) $\beta>0, t>1$ or $\beta>0, t=1$ and $\lambda>\frac{2(\beta+s) \beta r}{(1-m) s}$.

Then, the speed tends to zero, and we have the following estimates as $k \rightarrow+\infty$ :

$$
\begin{aligned}
\text { (pointwise) } \quad f_{\lambda_{k}}\left(x_{k}\right)-\min f=\mathcal{O}\left(\frac{1}{k^{2}}\right), f\left(\operatorname{prox}_{\lambda_{k} f}\left(x_{k}\right)\right)-\min f=\mathcal{O}\left(\frac{1}{k^{2}}\right), \\
\left\|x_{k+1}-x_{k}\right\|=\mathcal{O}\left(\frac{1}{k}\right),\left\|x_{k}-\operatorname{prox}_{\lambda_{k} f}\left(x_{k}\right)\right\|=\mathcal{O}\left(\frac{\sqrt{\lambda_{k}}}{k}\right),\left\|\nabla f_{\lambda_{k}}\left(x_{k}\right)\right\|=\mathcal{O}\left(\frac{1}{k \sqrt{\lambda_{k}}}\right) . \\
\left(\text { summation) } \quad \sum_{k} k\left\|x_{k}-x_{k-1}\right\|^{2}<+\infty, \quad \sum_{k} k \lambda_{k}\left\|\nabla f_{\lambda_{k}}\left(x_{k}\right)\right\|^{2}<+\infty,\right. \\
\sum_{k} k\left(f_{\lambda_{k}}\left(x_{k}\right)-\min f\right)<+\infty, \quad \sum_{k} k^{2}\left\|\nabla f_{\lambda_{k}}\left(x_{k}\right)\right\|^{2}<+\infty .
\end{aligned}
$$


ii) For $\beta=0, t \geq 0$ or $\beta>0$ and $t>1$ we have the following convergence rates of the values

$$
f_{\lambda_{k}}\left(x_{k}\right)-\min f=o\left(\frac{1}{k^{2}}\right) \quad \text { and } f\left(\operatorname{prox}_{\lambda_{k} f}\left(x_{k}\right)\right)-\min f=o\left(\frac{1}{k^{2}}\right) \text { as } k \rightarrow+\infty .
$$

In addition, $\left\|x_{k}-x_{k-1}\right\|=o\left(\frac{1}{k}\right)$ and $\lim _{k \rightarrow+\infty} \frac{k}{\sqrt{\lambda_{k}}}\left\|\operatorname{prox}_{\lambda_{k} f}\left(x_{k}\right)-x_{k}\right\|=0$.

iii) For $\beta=0, t \in[0,2]$ or $\beta>0, t \in] 1,2],\left(x_{k}\right)$ and $\left(y_{k}\right)$ converge weakly to some $\hat{x} \in \operatorname{argmin} f$.

Proof. I. The discrete energy functions. Take $z \in \operatorname{argmin} f$. In each of the three cases (a)-(c), our Lyapunov analysis is based on a different energy function.

(a) Case $\beta=0$. For each $k \geq 1$, consider the discrete energy function as in the proof of Theorem 1.1, with $a=0$ (in accordance with (1.12)), and with $A_{\lambda_{k}}=\nabla f_{\lambda_{k}}$, that is,

$$
\mathcal{E}_{a, b}^{k}=\frac{1}{2}\left\|b\left(x_{k-1}-z\right)+t_{k}\left(x_{k}-x_{k-1}+s \nabla f_{\lambda_{k}}\left(x_{k}\right)\right)\right\|^{2}+\frac{b(1-b)}{2}\left\|x_{k-1}-z\right\|^{2} .
$$

Arguing as in the proof of Theorem 1.1, equation (1.18) in this particular instance becomes

$$
\begin{aligned}
\mathcal{E}_{a, b}^{k+1}-\mathcal{E}_{a, b}^{k}= & -b s t_{k}\left\langle\nabla f_{\lambda_{k}}\left(x_{k}\right), x_{k}-z\right\rangle-\frac{1}{2} s^{2} t_{k}^{2}\left\|\nabla f_{\lambda_{k}}\left(x_{k}\right)\right\|^{2} \\
& -s t_{k}\left(t_{k}-b\right)\left\langle\nabla f_{\lambda_{k}}\left(x_{k}\right), x_{k}-x_{k-1}\right\rangle+\frac{1}{2}(b-1)\left(2 t_{k}-1\right)\left\|x_{k}-x_{k-1}\right\|^{2} .
\end{aligned}
$$

By using successively the gradient inequality, and the fact that the function $\lambda \mapsto f_{\lambda}$ is non-increasing and the sequence $\left(\lambda_{k}\right)$ is non-decreasing we get

$$
\left\langle\nabla f_{\lambda_{k}}\left(x_{k}\right), x_{k-1}-x_{k}\right\rangle \leq f_{\lambda_{k}}\left(x_{k-1}\right)-f_{\lambda_{k}}\left(x_{k}\right) \leq f_{\lambda_{k-1}}\left(x_{k-1}\right)-f_{\lambda_{k}}\left(x_{k}\right) .
$$

Set $\epsilon:=\frac{-1+\sqrt{9-8 m}}{8}$ and $b:=m+\epsilon$. Since $0<m<1$, one can easily verify that $\epsilon>0$ and $0<b<1$. Since by assumption there exists $k_{1} \geq 0$ such that $1 \leq t_{k}$ for all $k \geq k_{1}$, we obtain

$$
\begin{aligned}
& -s t_{k}\left(t_{k}-b\right)\left\langle\nabla f_{\lambda_{k}}\left(x_{k}\right), x_{k}-x_{k-1}\right\rangle=s t_{k}\left(t_{k}-m-\epsilon\right)\left\langle\nabla f_{\lambda_{k}}\left(x_{k}\right), x_{k-1}-x_{k}\right\rangle \leq \\
& s t_{k}\left(t_{k}-m\right)\left(f_{\lambda_{k-1}}\left(x_{k-1}\right)-f_{\lambda_{k}}\left(x_{k}\right)\right)+\epsilon t_{k}\left\langle\nabla f_{\lambda_{k}}\left(x_{k}\right), x_{k}-x_{k-1}\right\rangle, \text { for all } k \geq k_{1} .
\end{aligned}
$$

Moreover, according to the gradient inequality, we have that, for all $k \geq 1$

$$
\begin{aligned}
-b s t_{k}\left\langle\nabla f_{\lambda_{k}}\left(x_{k}\right), x_{k}-z\right\rangle & =-(m+\epsilon) s t_{k}\left\langle\nabla f_{\lambda_{k}}\left(x_{k}\right), x_{k}-z\right\rangle \\
& \leq m s t_{k}\left(f_{\lambda_{k}}(z)-f_{\lambda_{k}}\left(x_{k}\right)\right)-\epsilon s t_{k}\left\langle\nabla f_{\lambda_{k}}\left(x_{k}\right), x_{k}-z\right\rangle \\
& =\operatorname{mst}_{k}\left(\min f-f_{\lambda_{k}}\left(x_{k}\right)\right)-\epsilon s t_{k}\left\langle\nabla f_{\lambda_{k}}\left(x_{k}\right), x_{k}-z\right\rangle .
\end{aligned}
$$

Now using the fact that $f_{\lambda_{k}}(z)-f_{\lambda_{k}}\left(x_{k}\right)=\left(f_{\lambda_{k}}(z)-\min f\right)-\left(f_{\lambda_{k}}\left(x_{k}\right)-\min f\right)$, and using (2.6) the last two relations give

$$
\begin{aligned}
& -b s t_{k}\left\langle\nabla f_{\lambda_{k}}\left(x_{k}\right), x_{k}-z\right\rangle-s t_{k}\left(t_{k}-b\right)\left\langle\nabla f_{\lambda_{k}}\left(x_{k}\right), x_{k}-x_{k-1}\right\rangle \\
& \leq s t_{k}\left(t_{k}-m\right)\left(f_{\lambda_{k-1}}\left(x_{k-1}\right)-\min f\right)-s t_{k}^{2}\left(f_{\lambda_{k}}\left(x_{k}\right)-\min f\right) \\
& \quad-\epsilon s t_{k}\left\langle\nabla f_{\lambda_{k}}\left(x_{k}\right), x_{k}-z\right\rangle+\epsilon s t_{k}\left\langle\nabla f_{\lambda_{k}}\left(x_{k}\right), x_{k}-x_{k-1}\right\rangle \\
& \leq s t_{k-1}^{2}\left(f_{\lambda_{k-1}}\left(x_{k-1}\right)-\min f\right)-s t_{k}^{2}\left(f_{\lambda_{k}}\left(x_{k}\right)-\min f\right) \\
& \quad-\epsilon s t_{k}\left\langle\nabla f_{\lambda_{k}}\left(x_{k}\right), x_{k}-z\right\rangle+\epsilon s t_{k}\left\langle\nabla f_{\lambda_{k}}\left(x_{k}\right), x_{k}-x_{k-1}\right\rangle, \text { for all } k \geq k_{1}+1 .
\end{aligned}
$$


Combining (2.8) and (2.11), we obtain, for all $k \geq k_{1}+1$

$$
\begin{aligned}
\mathcal{E}_{a, b}^{k+1}-\mathcal{E}_{a, b}^{k} & +s t_{k}^{2}\left(f_{\lambda_{k}}\left(x_{k}\right)-\min f\right)-s t_{k-1}^{2}\left(f_{\lambda_{k-1}}\left(x_{k-1}\right)-\min f\right) \\
& \leq-\epsilon s t_{k}\left\langle\nabla f_{\lambda_{k}}\left(x_{k}\right), x_{k}-z\right\rangle+\epsilon s t_{k}\left\langle\nabla f_{\lambda_{k}}\left(x_{k}\right), x_{k}-x_{k-1}\right\rangle \\
& -\frac{1}{2} s^{2} t_{k}^{2}\left\|\nabla f_{\lambda_{k}}\left(x_{k}\right)\right\|^{2}-\frac{1}{2}(1-b)\left(2 t_{k}-1\right)\left\|x_{k}-x_{k-1}\right\|^{2} .
\end{aligned}
$$

Take $p>\frac{\epsilon s}{4(1-b)}$, and write the elementary algebraic inequality

$$
\epsilon s t_{k}\left\langle\nabla f_{\lambda_{k}}\left(x_{k}\right), x_{k}-x_{k-1}\right\rangle \leq p \epsilon s t_{k}\left\|\nabla f_{\lambda_{k}}\left(x_{k}\right)\right\|^{2}+\frac{\epsilon s t_{k}}{4 p}\left\|x_{k}-x_{k-1}\right\|^{2} .
$$

Since $t_{k}=r k+q, r>0$, there exists $\epsilon_{1}>0, \epsilon_{2}>0$ and $k_{2} \geq k_{1}+1$ such that for all $k \geq k_{2}$

$$
p \epsilon s t_{k}-\frac{1}{2} s^{2} t_{k}^{2}<-\epsilon_{1} t_{k}^{2} \quad \text { and } \quad \frac{\epsilon s t_{k}}{4 p}-\frac{1}{2}(1-b)\left(2 t_{k}-1\right)<-\epsilon_{2} t_{k},
$$

where the last above inequality comes from the choice of $p$. Therefore,

$$
\begin{aligned}
& \epsilon s t_{k}\left\langle\nabla f_{\lambda_{k}}\left(x_{k}\right), x_{k}-x_{k-1}\right\rangle-\frac{1}{2} s^{2} t_{k}^{2}\left\|\nabla f_{\lambda_{k}}\left(x_{k}\right)\right\|^{2}-\frac{1}{2}(1-b)\left(2 t_{k}-1\right)\left\|x_{k}-x_{k-1}\right\|^{2} \\
& \leq-\epsilon_{1} t_{k}^{2}\left\|\nabla f_{\lambda_{k}}\left(x_{k}\right)\right\|^{2}-\epsilon_{2} t_{k}\left\|x_{k}-x_{k-1}\right\|^{2}, \text { for all } k \geq k_{2} .
\end{aligned}
$$

According to the $\lambda_{k}$-cocoerciveness of $\nabla f_{\lambda_{k}}, \nabla f_{\lambda_{k}}(z)=0$, and the gradient inequality, we have

$$
\left\langle\nabla f_{\lambda_{k}}\left(x_{k}\right), x_{k}-z\right\rangle \geq \frac{1}{2}\left(f_{\lambda_{k}}\left(x_{k}\right)-\min f\right)+\frac{\lambda_{k}}{2}\left\|\nabla f_{\lambda_{k}}\left(x_{k}\right)\right\|^{2}, \text { for all } k \geq k_{2} .
$$

Consequently, (2.12) becomes, for all $k \geq k_{2}$

$$
\mathcal{E}_{a, b}^{k+1}-\mathcal{E}_{a, b}^{k}+s t_{k}^{2}\left(f_{\lambda_{k}}\left(x_{k}\right)-\min f\right)-s t_{k-1}^{2}\left(f_{\lambda_{k-1}}\left(x_{k-1}\right)-\min f\right)
$$

$+\frac{\epsilon}{2} s t_{k}\left(f_{\lambda_{k}}\left(x_{k}\right)-\min f\right)+\frac{\epsilon}{2} s t_{k} \lambda_{k}\left\|\nabla f_{\lambda_{k}}\left(x_{k}\right)\right\|^{2}+\epsilon_{1} t_{k}^{2}\left\|\nabla f_{\lambda_{k}}\left(x_{k}\right)\right\|^{2}+\epsilon_{2} t_{k}\left\|x_{k}-x_{k-1}\right\|^{2} \leq 0$.

(b) Case $\beta>0$ and $t=0$. Then $\lambda_{k}=\lambda>0$. For each $k \geq 1$, consider the discrete energy function as in the proof of Theorem 1.1, with $A_{\lambda_{k}}=\nabla f_{\lambda}$, that is,

$\mathcal{E}_{a, b}^{k}=a t_{k-1}\left\langle\nabla f_{\lambda}\left(x_{k-1}\right), x_{k-1}-z\right\rangle+\frac{1}{2}\left\|b\left(x_{k-1}-z\right)+t_{k}\left(x_{k}-x_{k-1}+s \nabla f_{\lambda}\left(x_{k}\right)\right)\right\|^{2}+\frac{b(1-b)}{2}\left\|x_{k-1}-z\right\|^{2}$ and for $d>0$ (which will fixed later) set

$$
W_{a, b, d}^{k}:=\mathcal{E}_{a, b}^{k}+d k^{2}\left\|\nabla f_{\lambda}\left(x_{k-1}\right)\right\|^{2} .
$$

Arguing as in the proof of Theorem 1.1, the equation (1.18) in this particular instance becomes

$$
\begin{aligned}
\mathcal{E}_{a, b}^{k+1}-\mathcal{E}_{a, b}^{k} & =\left(a t_{k}-b\left(\beta t_{k+1}+s t_{k}\right)\right)\left\langle\nabla f_{\lambda}\left(x_{k}\right), x_{k}-z\right\rangle+\left(b \beta t_{k+1}-a t_{k-1}\right)\left\langle\nabla f_{\lambda}\left(x_{k-1}\right), x_{k-1}-z\right\rangle \\
& +\frac{1}{2}\left(\beta^{2} t_{k+1}^{2}-s^{2} t_{k}^{2}\right)\left\|\nabla f_{\lambda}\left(x_{k}\right)\right\|^{2}-\beta^{2} t_{k+1}^{2}\left\langle\nabla f_{\lambda}\left(x_{k}\right), \nabla f_{\lambda}\left(x_{k-1}\right)\right\rangle+\frac{1}{2} \beta^{2} t_{k+1}^{2}\left\|\nabla f_{\lambda}\left(x_{k-1}\right)\right\|^{2} \\
& -\left(\beta t_{k+1}\left(t_{k}-1\right)+s t_{k}\left(t_{k}-b\right)\right)\left\langle\nabla f_{\lambda}\left(x_{k}\right), x_{k}-x_{k-1}\right\rangle \\
& +\beta t_{k+1}\left(t_{k}+b-1\right)\left\langle\nabla f_{\lambda}\left(x_{k-1}\right), x_{k}-x_{k-1}\right\rangle+\frac{1}{2}(b-1)\left(2 t_{k}-1\right)\left\|x_{k}-x_{k-1}\right\|^{2} .
\end{aligned}
$$


Therefore

$$
\begin{aligned}
& W_{a, b, d}^{k+1}-W_{a, b, d}^{k}=\left(a t_{k}-b\left(\beta t_{k+1}+s t_{k}\right)\right)\left\langle\nabla f_{\lambda}\left(x_{k}\right), x_{k}-z\right\rangle+\left(b \beta t_{k+1}-a t_{k-1}\right)\left\langle\nabla f_{\lambda}\left(x_{k-1}\right), x_{k-1}-z\right\rangle \\
&+ \frac{1}{2}\left(\beta^{2} t_{k+1}^{2}-s^{2} t_{k}^{2}+2 d(k+1)^{2}\right)\left\|\nabla f_{\lambda}\left(x_{k}\right)\right\|^{2}-\beta^{2} t_{k+1}^{2}\left\langle\nabla f_{\lambda}\left(x_{k}\right), \nabla f_{\lambda}\left(x_{k-1}\right)\right\rangle \\
&+ \frac{1}{2}\left(\beta^{2} t_{k+1}^{2}-2 d k^{2}\right)\left\|\nabla f_{\lambda}\left(x_{k-1}\right)\right\|^{2}-\left(\beta t_{k+1}\left(t_{k}-1\right)+s t_{k}\left(t_{k}-b\right)\right)\left\langle\nabla f_{\lambda}\left(x_{k}\right), x_{k}-x_{k-1}\right\rangle \\
&(2.16) \quad+\beta t_{k+1}\left(t_{k}+b-1\right)\left\langle\nabla f_{\lambda}\left(x_{k-1}\right), x_{k}-x_{k-1}\right\rangle+\frac{1}{2}(b-1)\left(2 t_{k}-1\right)\left\|x_{k}-x_{k-1}\right\|^{2} .
\end{aligned}
$$

According to the monotonicity of $\nabla f_{\lambda}$ and the assumption $t_{k}+b-1>0$ for all $k \geq k_{1}$, we have

$$
\begin{aligned}
-\left(\beta t_{k+1}\left(t_{k}-1\right)+s t_{k}\left(t_{k}-b\right)\right)\left\langle\nabla f_{\lambda}\left(x_{k}\right), x_{k}-x_{k-1}\right\rangle+\beta t_{k+1}\left(t_{k}+b-1\right)\left\langle\nabla f_{\lambda}\left(x_{k-1}\right), x_{k}-x_{k-1}\right\rangle \\
\left.\quad \leq\left(\beta b t_{k+1}-s t_{k}\left(t_{k}-b\right)\right)\right)\left\langle\nabla f_{\lambda}\left(x_{k}\right), x_{k}-x_{k-1}\right\rangle \\
\left.\quad=\left(\beta b t_{k+1}+s b t_{k}\right)\right)\left\langle\nabla f_{\lambda}\left(x_{k}\right), x_{k}-x_{k-1}\right\rangle+s t_{k}^{2}\left\langle\nabla f_{\lambda}\left(x_{k}\right), x_{k-1}-x_{k}\right\rangle .
\end{aligned}
$$

According to the gradient inequality we have, for all $k \geq k_{1}$

$$
\begin{aligned}
& s t_{k}^{2}\left\langle\nabla f_{\lambda}\left(x_{k}\right), x_{k-1}-x_{k}\right\rangle \leq s t_{k}^{2}\left(\left(f_{\lambda}\left(x_{k-1}\right)-\min f\right)-\left(f_{\lambda}\left(x_{k}\right)-\min f\right)\right) \\
& =s t_{k-1}^{2}\left(f_{\lambda}\left(x_{k-1}\right)-\min f\right)-s t_{k}^{2}\left(f_{\lambda}\left(x_{k}\right)-\min f\right)+s\left(t_{k}^{2}-t_{k-1}^{2}\right)\left(f_{\lambda}\left(x_{k-1}\right)-\min f\right) .
\end{aligned}
$$

Combining (2.17) and (2.18) with (2.16), we obtain

$$
\begin{aligned}
& W_{a, b, d}^{k+1}-W_{a, b, d}^{k}+s t_{k}^{2}\left(f_{\lambda}\left(x_{k}\right)-\min f\right)-s t_{k-1}^{2}\left(f_{\lambda}\left(x_{k-1}\right)-\min f\right) \\
& \leq\left(a t_{k}-b\left(\beta t_{k+1}+s t_{k}\right)\right)\left\langle\nabla f_{\lambda}\left(x_{k}\right), x_{k}-z\right\rangle+\left(b \beta t_{k+1}-a t_{k-1}\right)\left\langle\nabla f_{\lambda}\left(x_{k-1}\right), x_{k-1}-z\right\rangle \\
& \quad+\frac{1}{2}\left(\beta^{2} t_{k+1}^{2}-s^{2} t_{k}^{2}+2 d(k+1)^{2}\right)\left\|\nabla f_{\lambda}\left(x_{k}\right)\right\|^{2}-\beta^{2} t_{k+1}^{2}\left\langle\nabla f_{\lambda}\left(x_{k}\right), \nabla f_{\lambda}\left(x_{k-1}\right)\right\rangle \\
& \left.\quad+\frac{1}{2}\left(\beta^{2} t_{k+1}^{2}-2 d k^{2}\right)\left\|\nabla f_{\lambda}\left(x_{k-1}\right)\right\|^{2}+\left(\beta b t_{k+1}+s b t_{k}\right)\right)\left\langle\nabla f_{\lambda}\left(x_{k}\right), x_{k}-x_{k-1}\right\rangle \\
& \quad+\frac{1}{2}(b-1)\left(2 t_{k}-1\right)\left\|x_{k}-x_{k-1}\right\|^{2}+s\left(t_{k}^{2}-t_{k-1}^{2}\right)\left(f_{\lambda}\left(x_{k-1}\right)-\min f\right), \text { for all } k \geq k_{1} .
\end{aligned}
$$

Take $\beta b<a<b(\beta+s)$ and $0<b<1$. Since $r<\frac{1}{2}$, we can choose $a$ and $b$ satisfying the previous inequalities and such that there exists $k_{2} \geq k_{1}$ and $\epsilon_{3}>0$ such that

$$
a t_{k}-b\left(\beta t_{k+1}+s t_{k}\right)+\epsilon_{3} k \leq 0 \text { and } b \beta t_{k+1}-a t_{k-1}+s\left(t_{k}^{2}-t_{k-1}^{2}\right) \leq 0
$$

for all $k \geq k_{2}$ ( take $a=b \beta+(1-\epsilon) b s$ with $\epsilon$ sufficiently small, so that $\left.1>b>\frac{2 r}{1-\epsilon}\right)$. By using the gradient inequality

$$
\begin{aligned}
& e_{3} k\left(f_{\lambda}\left(x_{k}\right)-\min f\right) \leq \epsilon_{3} k\left\langle\nabla f_{\lambda}\left(x_{k}\right), x_{k}-z\right\rangle, \\
& \left(b \beta t_{k+1}-a t_{k-1}\right)\left\langle\nabla f_{\lambda}\left(x_{k-1}\right), x_{k-1}-z\right\rangle \leq\left(b \beta t_{k+1}-a t_{k-1}\right)\left(f_{\lambda}\left(x_{k-1}\right)-\min f\right), \text { for all } k \geq k_{2} .
\end{aligned}
$$

Therefore,

$$
\begin{aligned}
& \left(a t_{k}-b\left(\beta t_{k+1}+s t_{k}\right)+\epsilon_{3} k\right)\left\langle\nabla f_{\lambda}\left(x_{k}\right), x_{k}-z\right\rangle+\left(b \beta t_{k+1}-a t_{k-1}\right)\left\langle\nabla f_{\lambda}\left(x_{k-1}\right), x_{k-1}-z\right\rangle \\
& +s\left(t_{k}^{2}-t_{k-1}^{2}\right)\left(f_{\lambda}\left(x_{k-1}\right)-\min f\right) \leq 0, \text { for all } k \geq k_{2} .
\end{aligned}
$$


Combining (2.19) with (2.20), we get, for all $k \geq k_{2}$

$$
\begin{aligned}
& W_{a, b, d}^{k+1}-W_{a, b, d}^{k}+s t_{k}^{2}\left(f_{\lambda}\left(x_{k}\right)-\min f\right)-s t_{k-1}^{2}\left(f_{\lambda}\left(x_{k-1}\right)-\min f\right)+\epsilon_{3} k\left(f_{\lambda}\left(x_{k}\right)-\min f\right) \\
& \leq \frac{1}{2}\left(\beta^{2} t_{k+1}^{2}-s^{2} t_{k}^{2}+2 d(k+1)^{2}\right)\left\|\nabla f_{\lambda}\left(x_{k}\right)\right\|^{2}-\beta^{2} t_{k+1}^{2}\left\langle\nabla f_{\lambda}\left(x_{k}\right), \nabla f_{\lambda}\left(x_{k-1}\right)\right\rangle \\
& \left.+\frac{1}{2}\left(\beta^{2} t_{k+1}^{2}-2 d k^{2}\right)\left\|\nabla f_{\lambda}\left(x_{k-1}\right)\right\|^{2}+\left(\beta b t_{k+1}+s b t_{k}\right)\right)\left\langle\nabla f_{\lambda}\left(x_{k}\right), x_{k}-x_{k-1}\right\rangle \\
& +\frac{1}{2}(b-1)\left(2 t_{k}-1\right)\left\|x_{k}-x_{k-1}\right\|^{2} .
\end{aligned}
$$

We now use the following elementary algebraic inequalities

$$
\begin{aligned}
& -\beta^{2} t_{k+1}^{2}\left\langle\nabla f_{\lambda}\left(x_{k}\right), \nabla f_{\lambda}\left(x_{k-1}\right)\right\rangle \leq \frac{\beta^{2} t_{k+1}^{2}}{2}\left(\left\|\nabla f_{\lambda}\left(x_{k}\right)\right\|^{2}+\left\|\nabla f_{\lambda}\left(x_{k-1}\right)\right\|^{2}\right) \\
& \left(\beta b t_{k+1}+s b t_{k}\right)\left\langle\nabla f_{\lambda}\left(x_{k}\right), x_{k}-x_{k-1}\right\rangle \leq\left(\beta b t_{k+1}+s b t_{k}\right)\left(\frac{\sqrt{k}}{2}\left\|\nabla f_{\lambda}\left(x_{k}\right)\right\|^{2}+\frac{1}{2 \sqrt{k}}\left\|x_{k}-x_{k-1}\right\|^{2}\right) .
\end{aligned}
$$

Taking into account that $s>2 \beta$, we choose $d$ such that $\beta^{2} r^{2}<d<-\beta^{2} r^{2}+\frac{s^{2}}{2} r^{2}$.

Then, there exists $k_{3} \geq k_{2}$ and $\epsilon_{4}, \epsilon_{5}>0$ such that, for all $k \geq k_{3}$

$$
\begin{aligned}
& \frac{1}{2}\left(2 \beta^{2} t_{k+1}^{2}-s^{2} t_{k}^{2}+2 d(k+1)^{2}+\left(\beta b t_{k+1}+s b t_{k}\right) \sqrt{k}\right)+\epsilon_{4} k^{2} \leq 0 \\
& \beta^{2} t_{k+1}^{2}-d k^{2} \leq 0 \text { and } \frac{1}{2}\left((b-1)\left(2 t_{k}-1\right)+\frac{\beta b t_{k+1}+s b t_{k}}{\sqrt{k}}\right)+\epsilon_{5} k \leq 0
\end{aligned}
$$

Consequently, (2.21) becomes, for all $k \geq k_{3}$

$$
\begin{array}{r}
(2.22) \quad \begin{array}{r}
W_{a, b, d}^{k+1}-W_{a, b, d}^{k}+s t_{k}^{2}\left(f_{\lambda}\left(x_{k}\right)-\min f\right)-s t_{k-1}^{2}\left(f_{\lambda}\left(x_{k-1}\right)-\min f\right)+\epsilon_{3} k\left(f_{\lambda}\left(x_{k}\right)-\min f\right) \\
+\epsilon_{4} k^{2}\left\|\nabla f_{\lambda}\left(x_{k}\right)\right\|^{2}+\epsilon_{5} k\left\|x_{k}-x_{k-1}\right\|^{2}
\end{array} \\
\leq \frac{1}{2}\left(2 \beta^{2} t_{k+1}^{2}-s^{2} t_{k}^{2}+2 d(k+1)^{2}+\left(\beta b t_{k+1}+s b t_{k}\right) \sqrt{k}+2 \epsilon_{4} k^{2}\right)\left\|\nabla f_{\lambda}\left(x_{k}\right)\right\|^{2} \\
+\left(\beta^{2} t_{k+1}^{2}-d k^{2}\right)\left\|\nabla f_{\lambda}\left(x_{k-1}\right)\right\|^{2}+\frac{1}{2}\left((b-1)\left(2 t_{k}-1\right)+\frac{\beta b t_{k+1}+s b t_{k}}{\sqrt{k}}+2 \epsilon_{5} k\right)\left\|x_{k}-x_{k-1}\right\|^{2} \leq 0
\end{array}
$$

(c) Case $\beta>0$ and $t \geq 1$. For each $k \geq 1$, consider the discrete energy function as in the proof of Theorem 1.1, with $A_{\lambda_{k}}=\nabla f_{\lambda_{k}}$, that is,

$\mathcal{E}_{a, b}^{k}=a t_{k-1}\left\langle\nabla f_{\lambda_{k-1}}\left(x_{k-1}\right), x_{k-1}-z\right\rangle+\frac{1}{2}\left\|b\left(x_{k-1}-z\right)+t_{k}\left(x_{k}-x_{k-1}+s \nabla f_{\lambda_{k}}\left(x_{k}\right)\right)\right\|^{2}+\frac{b(1-b)}{2}\left\|x_{k-1}-z\right\|^{2}$.

Arguing as in the proof of Theorem 1.1, the equation (1.18) in this particular instance becomes

$$
\begin{aligned}
& \mathcal{E}_{a, b}^{k+1}-\mathcal{E}_{a, b}^{k}=\left(a t_{k}-b\left(\beta t_{k+1}+s t_{k}\right)\right)\left\langle\nabla f_{\lambda_{k}}\left(x_{k}\right), x_{k}-z\right\rangle+\left(b \beta t_{k+1}-a t_{k-1}\right)\left\langle\nabla f_{\lambda_{k-1}}\left(x_{k-1}\right), x_{k-1}-z\right\rangle \\
& +\frac{1}{2}\left(\beta^{2} t_{k+1}^{2}-s^{2} t_{k}^{2}\right)\left\|\nabla f_{\lambda_{k}}\left(x_{k}\right)\right\|^{2}-\beta^{2} t_{k+1}^{2}\left\langle\nabla f_{\lambda_{k}}\left(x_{k}\right), \nabla f_{\lambda_{k-1}}\left(x_{k-1}\right)\right\rangle+\frac{1}{2} \beta^{2} t_{k+1}^{2}\left\|\nabla f_{\lambda_{k-1}}\left(x_{k-1}\right)\right\|^{2} \\
& -\left(\beta t_{k+1}\left(t_{k}-1\right)+s t_{k}\left(t_{k}-b\right)\right)\left\langle\nabla f_{\lambda_{k}}\left(x_{k}\right), x_{k}-x_{k-1}\right\rangle \\
& +\beta t_{k+1}\left(t_{k}+b-1\right)\left\langle\nabla f_{\lambda_{k-1}}\left(x_{k-1}\right), x_{k}-x_{k-1}\right\rangle+\frac{1}{2}(b-1)\left(2 t_{k}-1\right)\left\|x_{k}-x_{k-1}\right\|^{2}, \text { for all } k \geq 1 .
\end{aligned}
$$


From [13, Lemma A4], for all $k \geq 1$ we have

$$
\left\|\lambda_{k} \nabla f_{\lambda_{k}}\left(x_{k}\right)-\lambda_{k-1} \nabla f_{\lambda_{k-1}}\left(x_{k-1}\right)\right\| \leq 2\left\|x_{k}-x_{k-1}\right\|+\left|\lambda_{k}-\lambda_{k-1}\right|\left\|\nabla f_{\lambda_{k}}\left(x_{k}\right)\right\| .
$$

Hence, we have for all $k \geq 1$ that

$$
\begin{aligned}
& \left\langle\nabla f_{\lambda_{k-1}}\left(x_{k-1}\right), x_{k}-x_{k-1}\right\rangle=\frac{1}{\lambda_{k-1}}\left\langle\lambda_{k-1} \nabla f_{\lambda_{k-1}}\left(x_{k-1}\right)-\lambda_{k} \nabla f_{\lambda_{k}}\left(x_{k}\right), x_{k}-x_{k-1}\right\rangle \\
& +\frac{\lambda_{k}}{\lambda_{k-1}}\left\langle\nabla f_{\lambda_{k}}\left(x_{k}\right), x_{k}-x_{k-1}\right\rangle \leq \frac{1}{\lambda_{k-1}}\left\|\lambda_{k-1} \nabla f_{\lambda_{k-1}}\left(x_{k-1}\right)-\lambda_{k} \nabla f_{\lambda_{k}}\left(x_{k}\right)\right\|\left\|x_{k}-x_{k-1}\right\| \\
& +\frac{\lambda_{k}}{\lambda_{k-1}}\left\langle\nabla f_{\lambda_{k}}\left(x_{k}\right), x_{k}-x_{k-1}\right\rangle \\
& \leq \frac{2}{\lambda_{k-1}}\left\|x_{k}-x_{k-1}\right\|^{2}+\frac{\left|\lambda_{k}-\lambda_{k-1}\right|}{\lambda_{k-1}}\left\|\nabla f_{\lambda_{k}}\left(x_{k}\right)\right\|\left\|x_{k}-x_{k-1}\right\|+\frac{\lambda_{k}}{\lambda_{k-1}}\left\langle\nabla f_{\lambda_{k}}\left(x_{k}\right), x_{k}-x_{k-1}\right\rangle .
\end{aligned}
$$

Moreover, for every $p_{1}>0$ we have

$$
\left\|\nabla f_{\lambda_{k}}\left(x_{k}\right)\right\|\left\|x_{k}-x_{k-1}\right\| \leq p_{1} \sqrt{k}\left\|\nabla f_{\lambda_{k}}\left(x_{k}\right)\right\|^{2}+\frac{1}{4 p_{1} \sqrt{k}}\left\|x_{k}-x_{k-1}\right\|^{2} .
$$

Therefore, (2.24) becomes, for all $k \geq 1$

$$
\begin{aligned}
& \left\langle\nabla f_{\lambda_{k-1}}\left(x_{k-1}\right), x_{k}-x_{k-1}\right\rangle \leq \frac{\lambda_{k}}{\lambda_{k-1}}\left\langle\nabla f_{\lambda_{k}}\left(x_{k}\right), x_{k}-x_{k-1}\right\rangle \\
& +\left(\frac{2}{\lambda_{k-1}}+\frac{\left|\lambda_{k}-\lambda_{k-1}\right|}{4 p_{1} \sqrt{k} \lambda_{k-1}}\right)\left\|x_{k}-x_{k-1}\right\|^{2}+\frac{p_{1} \sqrt{k}\left|\lambda_{k}-\lambda_{k-1}\right|}{\lambda_{k-1}}\left\|\nabla f_{\lambda_{k}}\left(x_{k}\right)\right\|^{2} .
\end{aligned}
$$

Combining the above results, we obtain (we write shortly $\Delta_{k}=\mathcal{E}_{a, b}^{k+1}-\mathcal{E}_{a, b}^{k}$ ), for all $k \geq 1$,

$$
\begin{aligned}
\Delta_{k} \leq & \left(a t_{k}-b\left(\beta t_{k+1}+s t_{k}\right)\right)\left\langle\nabla f_{\lambda_{k}}\left(x_{k}\right), x_{k}-z\right\rangle+\left(b \beta t_{k+1}-a t_{k-1}\right)\left\langle\nabla f_{\lambda_{k-1}}\left(x_{k-1}\right), x_{k-1}-z\right\rangle \\
& +\frac{1}{2}\left(\beta^{2} t_{k+1}^{2}-s^{2} t_{k}^{2}+2 \beta t_{k+1}\left(t_{k}+b-1\right) \frac{p_{1} \sqrt{k}\left|\lambda_{k}-\lambda_{k-1}\right|}{\lambda_{k-1}}\right)\left\|\nabla f_{\lambda_{k}}\left(x_{k}\right)\right\|^{2} \\
& -\beta^{2} t_{k+1}^{2}\left\langle\nabla f_{\lambda_{k}}\left(x_{k}\right), \nabla f_{\lambda_{k-1}}\left(x_{k-1}\right)\right\rangle+\frac{1}{2} \beta^{2} t_{k+1}^{2}\left\|\nabla f_{\lambda_{k-1}}\left(x_{k-1}\right)\right\|^{2} \\
& +\left(\beta t_{k+1}\left(t_{k}-1\right) \frac{\lambda_{k}-\lambda_{k-1}}{\lambda_{k-1}}+\beta b t_{k+1} \frac{\lambda_{k}}{\lambda_{k-1}}-s t_{k}\left(t_{k}-b\right)\right)\left\langle\nabla f_{\lambda_{k}}\left(x_{k}\right), x_{k}-x_{k-1}\right\rangle \\
& +\left(\beta t_{k+1}\left(t_{k}+b-1\right)\left(\frac{2}{\lambda_{k-1}}+\frac{\left|\lambda_{k}-\lambda_{k-1}\right|}{4 p_{1} \sqrt{k} \lambda_{k-1}}\right)+\frac{1}{2}(b-1)\left(2 t_{k}-1\right)\right)\left\|x_{k}-x_{k-1}\right\|^{2} .
\end{aligned}
$$

Further estimates give

$$
\begin{aligned}
& -\beta^{2} t_{k+1}^{2}\left\langle\nabla f_{\lambda_{k}}\left(x_{k}\right), \nabla f_{\lambda_{k-1}}\left(x_{k-1}\right)\right\rangle \leq \frac{\beta^{2} t_{k+1}^{2}}{2}\left(\left\|\nabla f_{\lambda_{k}}\left(x_{k}\right)\right\|^{2}+\left\|\nabla f_{\lambda_{k-1}}\left(x_{k-1}\right)\right\|^{2}\right) \\
& \quad\left(\beta t_{k+1}\left(t_{k}-1\right) \frac{\lambda_{k}-\lambda_{k-1}}{\lambda_{k-1}}+\beta b t_{k+1} \frac{\lambda_{k}}{\lambda_{k-1}}+s b t_{k}\right)\left\langle\nabla f_{\lambda_{k}}\left(x_{k}\right), x_{k}-x_{k-1}\right\rangle \\
& \leq \\
& \left(\beta t_{k+1}\left(t_{k}-1\right) \frac{\lambda_{k}-\lambda_{k-1}}{\lambda_{k-1}}+\beta b t_{k+1} \frac{\lambda_{k}}{\lambda_{k-1}}+s b t_{k}\right)\left(\sqrt{k}\left\|\nabla f_{\lambda_{k}}\right\|^{2}+\frac{1}{4 \sqrt{k}}\left\|x_{k}-x_{k-1}\right\|^{2}\right) .
\end{aligned}
$$


To simplify the formulation of the formulas, let us denote

$r_{1}(k)=-\frac{1}{2}(b-1)+\frac{2 \beta(b-1) t_{k+1}}{\lambda_{k-1}}+\beta t_{k+1}\left(t_{k}+b-1\right) \frac{\left|\lambda_{k}-\lambda_{k-1}\right|}{4 p_{1} \sqrt{k} \lambda_{k-1}}$,

$r_{2}(k)=\left(\left(\beta t_{k+1}\left(t_{k}-1\right) \frac{\lambda_{k}-\lambda_{k-1}}{\lambda_{k-1}}+\beta b t_{k+1} \frac{\lambda_{k}}{\lambda_{k-1}}+s b t_{k}\right) \frac{1}{4 \sqrt{k}}\right.$

$r_{3}(k)=\left(\left(\beta t_{k+1}\left(t_{k}-1\right) \frac{\lambda_{k}-\lambda_{k-1}}{\lambda_{k-1}}+\beta b t_{k+1} \frac{\lambda_{k}}{\lambda_{k-1}}+s b t_{k}\right) \sqrt{k}+2 \beta t_{k+1}\left(t_{k}+b-1\right) \frac{p_{1} \sqrt{k}\left|\lambda_{k}-\lambda_{k-1}\right|}{\lambda_{k-1}}\right.$.

Since $\lim _{x \rightarrow+\infty} \frac{x^{t}-(x-1)^{t}}{(x-1)^{t-1}}=t$, we have $\frac{\lambda_{k}-\lambda_{k-1}}{\lambda_{k-1}}=\mathcal{O}\left(\frac{1}{k}\right)$ as $k \rightarrow+\infty$. Hence,

$$
r_{1}(k)=\mathcal{O}\left(k^{\frac{1}{2}}\right), r_{2}(k)=\mathcal{O}\left(k^{\frac{1}{2}}\right), r_{3}(k)=\mathcal{O}\left(k^{\frac{3}{2}}\right), k \rightarrow+\infty .
$$

Consequently, (2.26), (2.27) and (2.28) yield, for all $k \geq 1$

$$
\begin{aligned}
\Delta_{k} & \leq\left(a t_{k}-b\left(\beta t_{k+1}+s t_{k}\right)\right)\left\langle\nabla f_{\lambda_{k}}\left(x_{k}\right), x_{k}-z\right\rangle+\left(b \beta t_{k+1}-a t_{k-1}\right)\left\langle\nabla f_{\lambda_{k-1}}\left(x_{k-1}\right), x_{k-1}-z\right\rangle \\
& +\frac{1}{2}\left(2 \beta^{2} t_{k+1}^{2}-s^{2} t_{k}^{2}+2 r_{3}(k)\right)\left\|\nabla f_{\lambda_{k}}\left(x_{k}\right)\right\|^{2}+\beta^{2} t_{k+1}^{2}\left\|\nabla f_{\lambda_{k-1}}\left(x_{k-1}\right)\right\|^{2} \\
(2.30) & -s t_{k}^{2}\left\langle\nabla f_{\lambda_{k}}\left(x_{k}\right), x_{k}-x_{k-1}\right\rangle+\left((b-1) t_{k}+\frac{2 \beta t_{k+1} t_{k}}{\lambda_{k-1}}+r_{1}(k)+r_{2}(k)\right)\left\|x_{k}-x_{k-1}\right\|^{2} .
\end{aligned}
$$

Now, using (2.18) we get, for all $k \geq k_{1}$

$$
\begin{aligned}
(2.31) & \Delta_{k}+s t_{k}^{2}\left(f_{\lambda}\left(x_{k}\right)-\min f\right)-s t_{k-1}^{2}\left(f_{\lambda}\left(x_{k-1}\right)-\min f\right) \\
& \leq\left(a t_{k}-b\left(\beta t_{k+1}+s t_{k}\right)\right)\left\langle\nabla f_{\lambda_{k}}\left(x_{k}\right), x_{k}-z\right\rangle+\left(b \beta t_{k+1}-a t_{k-1}\right)\left\langle\nabla f_{\lambda_{k-1}}\left(x_{k-1}\right), x_{k-1}-z\right\rangle \\
& +\frac{1}{2}\left(2 \beta^{2} t_{k+1}^{2}-s^{2} t_{k}^{2}+2 r_{3}(k)\right)\left\|\nabla f_{\lambda_{k}}\left(x_{k}\right)\right\|^{2}+\beta^{2} t_{k+1}^{2}\left\|\nabla f_{\lambda_{k-1}}\left(x_{k-1}\right)\right\|^{2} \\
& +s\left(t_{k}^{2}-t_{k-1}^{2}\right)\left(f_{\lambda}\left(x_{k-1}\right)-\min f\right)+\left((b-1) t_{k}+\frac{2 \beta t_{k+1} t_{k}}{\lambda_{k-1}}+r_{1}(k)+r_{2}(k)\right)\left\|x_{k}-x_{k-1}\right\|^{2} .
\end{aligned}
$$

Further, by the gradient inequality and the fact that $t_{k}^{2}-t_{k-1}^{2} \leq m t_{k}$ for all $k \geq k_{1}+1$ we have

$$
\begin{gathered}
\left(b \beta t_{k+1}-a t_{k-1}\right)\left\langle\nabla f_{\lambda_{k-1}}\left(x_{k-1}\right), x_{k-1}-z\right\rangle+s\left(t_{k}^{2}-t_{k-1}^{2}\right)\left(f_{\lambda}\left(x_{k-1}\right)-\min f\right) \\
\leq\left(b \beta t_{k+1}-a t_{k-1}+m s t_{k}\right)\left\langle\nabla f_{\lambda_{k-1}}\left(x_{k-1}\right), x_{k-1}-z\right\rangle, \text { for all } k \geq k_{1}+1 .
\end{gathered}
$$

Assume that $t=1$ and let $b=\frac{\beta+m s}{\beta+s} \in(m, 1)$ and $a=\beta b+\frac{(m+b) s}{2} \in(\beta b+m s, \beta b+b s)$.

Since by assumption we have $\lambda>\frac{2(\beta+s) \beta r}{(1-m) s}$ we conclude that there exist $\epsilon_{6}, \epsilon_{7}>0$ such that

$$
\begin{aligned}
& \left((a-\beta b-b s) r+\epsilon_{6}\right) \lambda+\frac{1}{2}\left(2 \beta^{2} r^{2}-s^{2} r^{2}\right)<0 \\
& (b-1) r+\frac{2 \beta r^{2}}{\lambda}+\epsilon_{7}<0 \text { and } \\
& (\beta b r-a r+m s r) \lambda+\beta^{2} r^{2}<0 .
\end{aligned}
$$


Assume that $t>1$ and fix $b \in(m, 1), a \in(\beta b+m s, \beta b+b s)$. Then take $\epsilon_{6}, \epsilon_{7}>0$ such that

$$
\begin{aligned}
& (a-\beta b-b s) r+\epsilon_{6}<0 \text { and } \\
& (b-1) r+\epsilon_{7}<0 .
\end{aligned}
$$

From now on, we do not need to distinguish the cases $t=1$ and $t>1$. According to the $\lambda_{k}$ cocoerciveness of $\nabla f_{\lambda_{k}},(2.32),(2.33),(2.29)$ and $t \geq 1$, we deduce that

$$
\begin{aligned}
& \left(a t_{k}-b\left(\beta t_{k+1}+s t_{k}\right)+\epsilon_{6} k\right)\left\langle\nabla f_{\lambda_{k}}\left(x_{k}\right), x_{k}-z\right\rangle+\frac{1}{2}\left(2 \beta^{2} t_{k+1}^{2}-s^{2} t_{k}^{2}+2 r_{3}(k)\right)\left\|\nabla f_{\lambda_{k}}\left(x_{k}\right)\right\|^{2} \leq 0, \\
& \left(b \beta t_{k+1}-a t_{k-1}+m s t_{k}\right)\left\langle\nabla f_{\lambda_{k-1}}\left(x_{k-1}\right), x_{k-1}-z\right\rangle+\beta^{2} t_{k+1}^{2}\left\|\nabla f_{\lambda_{k-1}}\left(x_{k-1}\right)\right\|^{2} \leq 0, \\
& \left((b-1) t_{k}+\frac{2 \beta t_{k+1} t_{k}}{\lambda_{k-1}}+r_{1}(k)+r_{2}(k)+\epsilon_{7} k\right)\left\|x_{k}-x_{k-1}\right\|^{2} \leq 0
\end{aligned}
$$

holds for some for $k_{2} \geq k_{1}+1$, and all $k \geq k_{2}$. Consequently, (2.31) leads to

$$
\begin{aligned}
& \Delta_{k}+s t_{k}^{2}\left(f_{\lambda}\left(x_{k}\right)-\min f\right)-s t_{k-1}^{2}\left(f_{\lambda}\left(x_{k-1}\right)-\min f\right) \\
& \leq-\epsilon_{6} k\left\langle\nabla f_{\lambda_{k}}\left(x_{k}\right), x_{k}-z\right\rangle-\epsilon_{7} k\left\|x_{k}-x_{k-1}\right\|^{2}, \text { for all } k \geq k_{2} .
\end{aligned}
$$

Then, use $-\epsilon_{6} k\left\langle\nabla f_{\lambda_{k}}\left(x_{k}\right), x_{k}-z\right\rangle \leq-\frac{\epsilon_{6}}{2} k \lambda_{k}\left\|\nabla f_{\lambda_{k}}\left(x_{k}\right)\right\|^{2}+\frac{\epsilon_{6}}{2} k\left(\min f-f_{\lambda_{k}}\left(x_{k}\right)\right)$, to finally obtain

$$
\begin{aligned}
& \Delta_{k}+s t_{k}^{2}\left(f_{\lambda}\left(x_{k}\right)-\min f\right)-s t_{k-1}^{2}\left(f_{\lambda}\left(x_{k-1}\right)-\min f\right) \\
& +\frac{\epsilon_{6}}{2} k \lambda_{k}\left\|\nabla f_{\lambda_{k}}\left(x_{k}\right)\right\|^{2}+\frac{\epsilon_{6}}{2} k\left(f_{\lambda_{k}}\left(x_{k}\right)-\min f\right)+\epsilon_{7} k\left\|x_{k}-x_{k-1}\right\|^{2} \leq 0, \text { for all } k \geq k_{2} .
\end{aligned}
$$

II. Estimates According to (2.14), (2.22), (2.35) the sequences of non-negative numbers

$$
\left(\mathcal{E}_{0, b}^{k}+t_{k}^{2}\left(f_{\lambda_{k}}\left(x_{k}\right)-\min f\right)\right)_{k},\left(W_{a, b, d}^{k}+t_{k}^{2}\left(f_{\lambda_{k}}\left(x_{k}\right)-\min f\right)\right)_{k} \text { and }\left(\mathcal{E}_{a, b}^{k}+t_{k}^{2}\left(f_{\lambda_{k}}\left(x_{k}\right)-\min f\right)\right)_{k}
$$

are non-increasing, and therefore converge. In particular, they are bounded. From this, and by adding the inequalities in (2.14), (2.22) and (2.35) we obtain:

$$
\begin{aligned}
& \sup _{k} k^{2}\left(f_{\lambda_{k}}\left(x_{k}\right)-\min f\right)<+\infty, \\
& \sup _{k}\left\|b\left(x_{k}-z\right)+t_{k+1}\left(x_{k+1}-x_{k}+s \nabla f_{\lambda_{k+1}}\left(x_{k+1}\right)\right)\right\|^{2}<+\infty, \\
& \sup _{k}\left\|x_{k}-z\right\|^{2}<+\infty, \\
& \sum_{k=0}^{+\infty} k\left(f_{\lambda_{k}}\left(x_{k}\right)-\min f\right)<+\infty, \\
& \sum_{k=0}^{+\infty} k \lambda_{k}\left\|\nabla f_{\lambda_{k}}\left(x_{k}\right)\right\|^{2}<+\infty, \\
& \sum_{k=0}^{+\infty} k^{2}\left\|\nabla f_{\lambda_{k}}\left(x_{k}\right)\right\|^{2}<+\infty, \\
& \sum_{k=1}^{+\infty} k\left\|x_{k}-x_{k-1}\right\|^{2}<+\infty .
\end{aligned}
$$


Obviously (2.36) assures that

$$
f_{\lambda_{k}}\left(x_{k}\right)-\min f=\mathcal{O}\left(\frac{1}{k^{2}}\right) \text { as } k \rightarrow+\infty .
$$

Since the general term of a convergent series goes to zero, we deduce from (2.41) that

$$
\left\|\nabla f_{\lambda_{k}}\left(x_{k}\right)\right\|=o\left(\frac{1}{k}\right) \text { as } k \rightarrow+\infty .
$$

The same argument applied to (2.40) yields

$$
\left\|\nabla f_{\lambda_{k}}\left(x_{k}\right)\right\|=o\left(\frac{1}{\sqrt{k \lambda_{k}}}\right) \text { as } k \rightarrow+\infty .
$$

Further, (2.38) shows that $\left\|x_{k}-z\right\|$ is bounded. Consequently, the sequence $\left(x_{k}\right)$ is bounded. Combining the above results with (2.37), we obtain

$$
\left\|x_{k}-x_{k-1}\right\|=\mathcal{O}\left(\frac{1}{k}\right) \text { as } k \rightarrow+\infty .
$$

From $f_{\lambda_{k}}\left(x_{k}\right)-\min f=f\left(\operatorname{prox}_{\lambda_{k} f}\left(x_{k}\right)\right)-\min f+\frac{1}{2 \lambda_{k}}\left\|x_{k}-\operatorname{prox}_{\lambda_{k} f}\left(x_{k}\right)\right\|^{2}$, we deduce that

$$
f\left(\operatorname{prox}_{\lambda_{k} f}\left(x_{k}\right)\right)-\min f=\mathcal{O}\left(\frac{1}{k^{2}}\right), \quad\left\|x_{k}-\operatorname{prox}_{\lambda_{k} f}\left(x_{k}\right)\right\|=\mathcal{O}\left(\frac{\sqrt{\lambda_{k}}}{k}\right) \text { as } k \rightarrow+\infty .
$$

Further we have $\nabla f_{\lambda_{k}}=(\partial f)_{\lambda_{k}}=\frac{1}{\lambda_{k}}\left(I-\operatorname{prox}_{\lambda_{k} f}\right)$, hence

$$
\left\|\nabla f_{\lambda_{k}}\left(x_{k}\right)\right\|=\mathcal{O}\left(\frac{1}{k \sqrt{\lambda_{k}}}\right) \text { as } k \rightarrow+\infty .
$$

III. The limit. Using the Opial's lemma, let us prove that the sequence $\left(x_{k}\right)$ generated by the algorithm (PRINAM)-convex converges weakly towards an element of $\operatorname{argmin} f$. Take $z \in \operatorname{argmin} f$, and consider the anchor sequence $\left(h_{k}\right)$ defined by $h_{k}=\frac{1}{2}\left\|x_{k}-z\right\|^{2}$ for $k \geq 1$. According to (1.38)

$$
h_{k+1}-h_{k}=\frac{1}{2}\left\|x_{k+1}-x_{k}\right\|^{2}+\left\langle x_{k+1}-x_{k}, x_{k}-z\right\rangle .
$$

According to the corresponding version of (1.6), we get

$$
\begin{aligned}
\left\langle x_{k+1}-x_{k}, x_{k}-z\right\rangle & =\left\langle y_{k}-x_{k}-s \nabla f_{\lambda_{k+1}}\left(x_{k+1}\right), x_{k}-z\right\rangle \\
& =\alpha_{k}\left\langle x_{k}-x_{k-1}, x_{k}-z\right\rangle-\beta\left\langle\nabla f_{\lambda_{k}}\left(x_{k}\right)-\nabla f_{\lambda_{k-1}}\left(x_{k-1}\right), x_{k}-z\right\rangle \\
& -s\left\langle\nabla f_{\lambda_{k+1}}\left(x_{k+1}\right), x_{k}-z\right\rangle .
\end{aligned}
$$

Let us examine the terms involved in the above equality. We have

$$
\begin{aligned}
& \left\langle x_{k}-x_{k-1}, x_{k}-z\right\rangle=\left\|x_{k}-x_{k-1}\right\|^{2}+\left\langle x_{k}-x_{k-1}, x_{k-1}-z\right\rangle=h_{k}-h_{k-1}+\frac{1}{2}\left\|x_{k}-x_{k-1}\right\|^{2} \\
& -s\left\langle\nabla f_{\lambda_{k+1}}\left(x_{k+1}\right), x_{k}-z\right\rangle=s\left\langle\nabla f_{\lambda_{k+1}}\left(x_{k+1}\right), x_{k+1}-x_{k}\right\rangle-s\left\langle\nabla f_{\lambda_{k+1}}\left(x_{k+1}\right), x_{k+1}-z\right\rangle .
\end{aligned}
$$


Combining these relations with (1.38) and (2.49), and neglecting the term $-s\left\langle\nabla f_{\lambda_{k+1}}\left(x_{k+1}\right), x_{k+1}-\right.$ $z\rangle$ which is non-positive, we obtain

$$
\begin{aligned}
h_{k+1}-h_{k} & \leq \alpha_{k}\left(h_{k}-h_{k-1}\right)+\frac{1}{2}\left\|x_{k+1}-x_{k}\right\|^{2}+\frac{\alpha_{k}}{2}\left\|x_{k}-x_{k-1}\right\|^{2} \\
& -\beta\left\langle\nabla f_{\lambda_{k}}\left(x_{k}\right)-\nabla f_{\lambda_{k-1}}\left(x_{k-1}\right), x_{k}-z\right\rangle+s\left\|\nabla f_{\lambda_{k+1}}\left(x_{k+1}\right)\right\|\left\|x_{k+1}-x_{k}\right\| .
\end{aligned}
$$

Since $\left\|x_{k}-z\right\|$ is bounded, we get the existence of a constant $M>0$ such that, for all $k \geq 1$

$$
\begin{aligned}
h_{k+1}-h_{k} & \leq \alpha_{k}\left(h_{k}-h_{k-1}\right)+\frac{1}{2}\left\|x_{k+1}-x_{k}\right\|^{2}+\frac{\alpha_{k}}{2}\left\|x_{k}-x_{k-1}\right\|^{2} \\
& +M \beta\left\|\nabla f_{\lambda_{k}}\left(x_{k}\right)-\nabla f_{\lambda_{k-1}}\left(x_{k-1}\right)\right\|+s\left\|\nabla f_{\lambda_{k+1}}\left(x_{k+1}\right)\right\|\left\|x_{k+1}-x_{k}\right\| .
\end{aligned}
$$

Further, when $\beta>0$, from Lemma [13, Lemma A4], we get

$$
\left\|\nabla f_{\lambda_{k}}\left(x_{k}\right)-\nabla f_{\lambda_{k-1}}\left(x_{k-1}\right)\right\| \leq \frac{2}{\lambda_{k}}\left\|x_{k}-x_{k-1}\right\|+\frac{\left|\lambda_{k}-\lambda_{k-1}\right|}{\lambda_{k}}\left(\left\|\nabla f_{\lambda_{k}}\left(x_{k}\right)\right\|+\left\|\nabla f_{\lambda_{k-1}}\left(x_{k-1}\right)\right\|\right) .
$$

Recall that $\lambda_{k}=\lambda k^{t}, t>1$ and $\frac{\lambda_{k}-\lambda_{k-1}}{\lambda_{k}}=\mathcal{O}\left(\frac{1}{k}\right)$ as $k \rightarrow+\infty$. Then, from (2.46) and (2.48) we obtain that there exists $\bar{k} \geq 1$ and $C>0$ such that, for all $k \geq \bar{k}$

$$
\begin{aligned}
& \frac{2}{\lambda_{k}}\left\|x_{k}-x_{k-1}\right\| \leq \frac{C}{k^{1+t}} \\
& \frac{\left|\lambda_{k}-\lambda_{k-1}\right|}{\lambda_{k}}\left(\left\|\nabla f_{\lambda_{k}}\left(x_{k}\right)\right\|+\left\|\nabla f_{\lambda_{k-1}}\left(x_{k-1}\right)\right\|\right) \leq \frac{C}{k^{2+\frac{t}{2}}} .
\end{aligned}
$$

Therefore, for all $k \geq \bar{k}$

$$
\left\|\nabla f_{\lambda_{k}}\left(x_{k}\right)-\nabla f_{\lambda_{k-1}}\left(x_{k-1}\right)\right\| \leq \frac{C}{k^{1+t}}+\frac{C}{k^{2+\frac{t}{2}}} .
$$

Consequently, (2.51) leads to

$$
h_{k+1}-h_{k} \leq \alpha_{k}\left(h_{k}-h_{k-1}\right)+\omega_{k}
$$

for all $k \geq \bar{k}$, where

$$
\omega_{k}=\left\{\begin{array}{l}
\frac{1}{2}\left\|x_{k+1}-x_{k}\right\|^{2}+\frac{\alpha_{k}}{2}\left\|x_{k}-x_{k-1}\right\|^{2}+s\left\|\nabla f_{\lambda_{k+1}}\left(x_{k+1}\right)\right\|\left\|x_{k+1}-x_{k}\right\|, \text { if } \beta=0 \\
\frac{1}{2}\left\|x_{k+1}-x_{k}\right\|^{2}+\frac{\alpha_{k}}{2}\left\|x_{k}-x_{k-1}\right\|^{2}+\beta M C\left(\frac{1}{k^{1+t}}+\frac{1}{k^{2+\frac{t}{2}}}\right)+s\left\|\nabla f_{\lambda_{k+1}}\left(x_{k+1}\right)\right\|\left\|x_{k+1}-x_{k}\right\|, \\
\text { if } \beta>0, t>1 .
\end{array}\right.
$$

As a direct consequence of the majorization

$$
\left\|\nabla f_{\lambda_{k+1}}\left(x_{k+1}\right)\right\|\left\|x_{k+1}-x_{k}\right\| \leq \frac{1}{2}\left\|\nabla f_{\lambda_{k+1}}\left(x_{k+1}\right)\right\|^{2}+\frac{1}{2}\left\|x_{k+1}-x_{k}\right\|^{2},
$$

of (2.42) and (2.41), and of the fact that $t>1$ if $\beta>0$, we have $\sum_{k} t_{k+1} \omega_{k}<+\infty$. Therefore, by applying Lemma A.1 to the sequence $a_{k}=\left[h_{k}-h_{k-1}\right]_{+}$we obtain

$$
\sum_{k}\left[h_{k}-h_{k-1}\right]_{+}<+\infty \text {. }
$$


Since $h_{k}$ is nonnegative, this property classically gives the existence of $\lim _{k \rightarrow+\infty} h_{k}$, and hence of the existence of $\lim _{k \rightarrow+\infty}\left\|x_{k}-z\right\|$. This shows item (i) of the Opial lemma.

Let us return to the fact that, according to (2.14) and (2.35), the sequences of non-negative numbers

$$
\left(\mathcal{E}_{0, b}^{k}+t_{k}^{2}\left(f_{\lambda_{k}}\left(x_{k}\right)-\min f\right)\right)_{k} \text { and }\left(\mathcal{E}_{a, b}^{k}+t_{k}^{2}\left(f_{\lambda_{k}}\left(x_{k}\right)-\min f\right)\right)_{k}
$$

are non-increasing, and therefore converge. Since $\left\|x_{k}-z\right\|$ converges, and $t_{k}\left\|\nabla f_{\lambda_{k}}\left(x_{k}\right)\right\| \rightarrow 0$, as $k \rightarrow+\infty$ we obtain that the following limit exists

$$
\lim _{k \rightarrow+\infty}\left(t_{k}^{2}\left\|x_{k}-x_{k-1}\right\|^{2}+t_{k}^{2}\left(f_{\lambda_{k}}\left(x_{k}\right)-\min f\right)\right) .
$$

On the other hand, according to (2.39) we have $\sum_{k=0}^{+\infty} t_{k}\left(f_{\lambda_{k}}\left(x_{k}\right)-\min f\right)<+\infty$, and according to (2.42) we have $\sum_{k=1}^{+\infty} t_{k}\left\|x_{k}-x_{k-1}\right\|^{2}<+\infty$. Therefore,

$$
\sum_{k=1}^{+\infty} \frac{1}{t_{k}}\left(t_{k}^{2}\left\|x_{k}-x_{k-1}\right\|^{2}+t_{k}^{2}\left(f_{\lambda_{k}}\left(x_{k}\right)-\min f\right)\right)<+\infty .
$$

Combining (2.54) and (2.55) we obtain that

$$
\lim _{k \rightarrow+\infty}\left(t_{k}^{2}\left\|x_{k}-x_{k-1}\right\|^{2}+t_{k}^{2}\left(f_{\lambda_{k}}\left(x_{k}\right)-\min f\right)\right)=0,
$$

that is, $\left\|x_{k}-x_{k-1}\right\|=o\left(\frac{1}{k}\right)$ and $f_{\lambda_{k}}\left(x_{k}\right)-\min f=o\left(\frac{1}{k^{2}}\right)$ as $k \rightarrow+\infty$.

From $f_{\lambda_{k}}\left(x_{k}\right)-\min f=f\left(\operatorname{prox}_{\lambda_{k} f}\left(x_{k}\right)\right)-\min f+\frac{1}{2 \lambda_{k}}\left\|x_{k}-\operatorname{prox}_{\lambda_{k} f}\left(x_{k}\right)\right\|^{2}$, we deduce that

$$
f\left(\operatorname{prox}_{\lambda_{k} f}\left(x_{k}\right)\right)-\min f=o\left(\frac{1}{k^{2}}\right), \quad \lim _{k \rightarrow+\infty} \frac{k^{2}}{\lambda_{k}}\left\|x_{k}-\operatorname{prox}_{\lambda_{k} f}\left(x_{k}\right)\right\|^{2}=0 .
$$

It remains to show that every weak cluster point of the sequence $\left(x_{k}\right)$ belongs to $\operatorname{argmin} f$. Let $x^{*}$ be a weak cluster point of $\left(x_{k}\right)$ and consider a subsequence $\left(x_{k_{n}}\right)$ of $\left(x_{k}\right)$, such that

$$
x_{k_{n}} \rightarrow x^{*}, n \rightarrow+\infty \text {. }
$$

If $t \leq 2$, then one has $\lim _{k \rightarrow+\infty}\left\|x_{k}-\operatorname{prox}_{\lambda_{k} f}\left(x_{k}\right)\right\|=0$. Therefore,

$$
\operatorname{prox}_{\lambda_{k_{n}} f}\left(x_{k_{n}}\right) \rightarrow x^{*}, n \rightarrow+\infty \text {. }
$$

Since $f$ is lower semi-continuous and convex, it is weakly lower semi-continuous. Combined with

$$
\lim _{k \rightarrow+\infty}\left(f\left(\operatorname{prox}_{\lambda_{k} f}\left(x_{k}\right)\right)-\min f\right)=0,
$$

it yields

$$
0=\liminf _{x_{k_{n}} \rightarrow x^{*}}\left(f\left(\operatorname{prox}_{\lambda_{k_{n}} f}\left(x_{k_{n}}\right)\right)-\min f\right) \geq f\left(x^{*}\right)-\min f .
$$

The latter relation shows that $x^{*} \in \operatorname{argmin} f$ and consequently, according to Opial lemma, the sequence $\left(x_{k}\right)$ converges weakly to an element $\hat{x} \in \operatorname{argmin} f$. Finally, since

$$
\left\|x_{k}-x_{k-1}\right\|=o\left(\frac{1}{k}\right),\left\|\nabla f_{\lambda_{k}}\left(x_{k}\right)-\nabla f_{\lambda_{k-1}}\left(x_{k-1}\right)\right\|=o\left(\frac{1}{k}\right), \text { as } k \rightarrow+\infty
$$

we get that

$$
\left\|x_{k}-y_{k}\right\|=o\left(\frac{1}{k}\right), \text { as } k \rightarrow+\infty,
$$

hence $y_{k}$ converges weakly to the same element $\hat{x} \in \operatorname{argmin} f$. 
3. Conclusion, Perspectives. (PRINAM) is a proximal-based inertial algorithm which aims to solve general monotone inclusions in Hilbert spaces. It has several favorable features:

1. Under the sole assumption that the solution set is nonempty, each sequence generated by the algorithm converges weakly to a zero of the operator.

2. The algorithm involves a correcting term which is naturally linked to the Hessian driven damping in the case of convex minimization, and to the Newton method for general monotone inclusions. There is numerical evidence of the fact that this correcting term attenuates the oscillations which naturally occur with the inertial methods.

3. When specializing the maximally monotone operator to the subdifferential of a convex lower semicontinuous proper function, the algorithm improves the accelerated gradient method of Nesterov by giving the convergence rate $o\left(\frac{1}{k^{2}}\right)$ of the values, and the fast convergence of the gradients towards zero.

The article presents the basic elements of the convergence theory for (PRINAM), many aspects of which have yet to be developed. We need to enlarge the framework by considering structured composite monotone inclusions, and show how to use (PRINAM) as the basic block of splitting algorithms such as (ADMM), Douglas-Rachford, to cite some of them. For numerical reasons, it is important to consider the introduction of perturbations, errors in the algorithms. Considering a Tikhonov regularization term with vanishing coefficient would allow to obtain strong convergence of the iterates towards the minimum norm solution, a desirable feature for the inverse problems. Various versions/extensions of (PRINAM) can also be considered, including the case of a variable stepsize, and time rescaling.

Appendix A. Auxiliary results. In our analysis of (PRINAM) we need the following result.

Lemma A.1. Let $\left(a_{k}\right)$ be a sequence of non-negative real numbers which satisfies: for all $k \geq 0$

$$
a_{k+1} \leq \alpha_{k} a_{k}+\omega_{k}
$$

where $\sum_{k} t_{k+1} \omega_{k}<+\infty$. Then $\sum_{k} a_{k}<+\infty$.

Proof. Since $\alpha_{k}=\frac{t_{k}-1}{t_{k+1}}$ we have $t_{k+1} a_{k+1}-\left(t_{k}-1\right) a_{k} \leq t_{k+1} \omega_{k}$. After summation, we obtain $t_{n+1} a_{n+1}-t_{0} a_{0}+\sum_{k=0}^{n} a_{k} \leq \sum_{k=0}^{n} t_{k+1} \omega_{k}$.

Hence, $\sum_{k=0}^{+\infty} a_{k} \leq \sum_{k=0}^{+\infty} t_{k+1} \omega_{k}+t_{0} a_{0}<+\infty$, which gives the claim.

\section{REFERENCES}

[1] B. Abbas, H. Aтtouch, B. F. Svaiter, Newton-like dynamics and forward-backward methods for structured monotone inclusions in Hilbert spaces, J. Optim. Theory Appl., 161 (2014), No. 2, pp. 331-360.

[2] F. Álvarez, H. Aтtouch, An inertial proximal method for maximal monotone operators via discretization of a nonlinear oscillator with damping, Set-Valued Analysis, 9 (2001), No. 1-2, pp. 3-11.

[3] F. Alvarez, H. Atтouch, J. Bolte, P. Redont, A second-order gradient-like dissipative dynamical system with Hessian-driven damping. Application to optimization and mechanics, J. Math. Pures Appl., 81(8) (2002), pp. 747-779.

[4] V. Apidopoulos, J.-F. Aujol, Ch. Dossal, Convergence rate of inertial Forward-Backward algorithm beyond Nesterov's rule, HAL-01551873, (2017), to appear in Math. Program.

[5] Н. Аттоuch, А. САвот, Convergence of a relaxed inertial proximal algorithm for maximally monotone operators, Math. Program. (2019). https://doi.org/10.1007/s10107-019-01412-0.

[6] Н. Аттоuch, А. Савот, Convergence rates of inertial forward-backward algorithms, SIAM J. Optim., 28 (2018), No. 1, pp. 849-874.

[7] H. Attouch, Z. Chbani, J. Fadili, H. Riahi, First-order algorithms via inertial systems with Hessian driven damping, (2019), HAL-02193846. 
[8] H. Attouch, Z. Chbani, J. Peypouquet, P. Redont, Fast convergence of inertial dynamics and algorithms with asymptotic vanishing viscosity, Math. Program. Ser. B 168 (2018), pp. 123-175.

[9] H. Aтtouch, Z. Chbani, H. Riahi, Rate of convergence of the Nesterov accelerated gradient method in the subcritical case $\alpha \leq 3$, arXiv:1706.05671v1 [math.OC] 2017, ESAIM-COCV (2019) published electronically, DOI $10.1051 / \mathrm{cocv} / 2017083$.

[10] H. Attouch, P.E. Maingé, Asymptotic behavior of second order dissipative evolution equations combining potential with non-potential effects, ESAIM Control Optim. Calc. of Var., 17 (2011), No. 3, pp. 836-857.

[11] H. Attouch, P.E. Maingé, P. Redont, A second-order differential system with Hessian-driven damping; Application to non-elastic shock laws, Differential Equations and Applications, 4 (2012), No. 1, pp. $27-65$.

[12] H. Attouch, M. Marques Alves, B. F. Svaiter, A dynamic approach to a proximal-Newton method for monotone inclusions in Hilbert Spaces, with complexity $\mathcal{O}\left(1 / n^{2}\right)$, Journal of Convex Analysis, 23 (2016), No. 1, pp. 139-180.

[13] H. Atтouch, J. Peypouquet, Convergence of inertial dynamics and proximal algorithms governed by maximal monotone operators, Mathematical Programming, 174 (1-2) (2019), pp. 391-432.

[14] H. Атtouch, J. Peypouquet, The rate of convergence of Nesterov's accelerated forward-backward method is actually faster than $1 / k^{2}$, SIAM J. Optim., 26 (2016), No. 3, pp. 1824-1834.

[15] H. Аtтouch, J. Peypouquet, P. Redont, Fast convex minimization via inertial dynamics with Hessian driven damping, J. Differential Equations, 261(10), (2016), pp. 5734-5783.

[16] H. Attouch, P. Redont, B. F. Svaiter, Global convergence of a closed-loop regularized Newton method for solving monotone inclusions in Hilbert spaces, J. Optim. Theory Appl., 157 (2013), No. 3, pp. 624-650.

[17] H. Atтouch, B. F. Svaiter, A continuous dynamical Newton-Like approach to solving monotone inclusions, SIAM J. Control Optim., 49 (2011), No. 2, pp. 574-598.

[18] H. Bauschke, P. L. Combettes, Convex Analysis and Monotone Operator Theory in Hilbert spaces, CMS Books in Mathematics, Springer, (2011).

[19] C. Castera, J. Bolte, C. Févotte, E. Pauwels, An Inertial Newton Algorithm for Deep Learning. 2019. HAL-02140748.

[20] R. I. Boţ, E. R. Csetnek, Second order forward-backward dynamical systems for monotone inclusion problems, SIAM J. Control Optim., 54 (2016), pp. 1423-1443.

[21] R. I. Boţ, E. R. Csetnek, S.C. LÁszló, Tikhonov regularization of a second order dynamical system with Hessian damping, arXiv:1911.12845v1 [math.OC] Nov 2019.

[22] A. Chambolle, Сн. Dossal, On the convergence of the iterates of the Fast Iterative Shrinkage Thresholding Algorithm, Journal of Optimization Theory and Applications, 166 (2015), pp. 968-982.

[23] P. L. Combettes, Monotone operator theory in convex optimization, Mathematical Programming, vol. B 170 (2018), no. 1, pp. 177-206.

[24] P. L. Combettes, L. Glaudin, Quasi-nonexpansive iterations on the affine hull of orbits: from Mann's mean value algorithm to inertial methods, SIAM J. Optim, 27 (2017), No. 4, pp. 2356-2380.

[25] F. Iutzeler, J. M. Hendrickx, A generic online acceleration scheme for optimization algorithms via relaxation and inertia, Optimization Methods and Software, 34 (2) (2019), pp. 383-405.

[26] D. Kim, Accelerated Proximal Point Method for Maximally Monotone Operators, preprint 6 Feb 2020, arXiv:1905.05149v3 [math.OC].

[27] T. Lin, M.I. Jordan, A Control-Theoretic Perspective on Optimal High-Order Optimization, (2019) arXiv:1912.07168v1 [math.OC] 16 Dec 2019.

[28] D.A. Lonenz, T. Pock, An inertial forward-backward algorithm for monotone inclusions, J. Math. Imaging and Vision, 51 (2015), pp. 311-325.

[29] R. MAY, Asymptotic for a second-order evolution equation with convex potential and vanishing damping term, Turkish Journal of Math., 41 (2017), No. 3, pp. 681-685.

[30] A. Moudafi, M. Oliny, Convergence of a splitting inertial proximal method for monotone operators, J. Comput. Appl. Math., 155 (2003), No. 2, pp. 447-454.

[31] B. SHI, S. S. Du, M. I. JoRdAn, W. J. Su, Understanding the acceleration phenomenon via high-resolution differential equations, arXiv:submit/2440124[cs.LG] 21 Oct 2018.

[32] W. J. Su, S. Boyd, E. J. CANDÈs, A differential equation for modeling Nesterov's accelerated gradient method: theory and insights. Neural Information Processing Systems 27 (2014), pp. 2510-2518. 